\title{
Degrees of Freedom of Rank-Deficient MIMO Interference Channels
}

\author{
Sundar R. Krishnamurthy, Student Member, IEEE, Abinesh Ramakrishnan, Student Member, IEEE, \\ and Syed Ali Jafar, Fellow, IEEE
}

\begin{abstract}
We characterize the degrees of freedom (DoF) of multiple-input and multiple-output (MIMO) interference channels with rank-deficient channel matrices. For the two-user rank-deficient MIMO interference channel, we provide a tight outer bound to show that the previously known achievable DoF in the symmetric case is optimal and generalize the result to fully asymmetric settings. For the $\boldsymbol{K}$-user rank-deficient interference channel, we improve the previously known achievable DoF and provide a tight outer bound to establish optimality in symmetric settings. In particular, we show that for the $K$-user rank-deficient interference channel, when all nodes have $M$ antennas, all direct channels have rank $D_{0}$, all cross channels are of rank $D$, and the channels are otherwise generic, the optimal DoF value per user is $\min \left(D_{0}, M-(\min (M,(K-1) D) / 2)\right)$. Notably for interference channels, the rank-deficiency of direct channels does not help and the rank deficiency of cross-channels does not hurt. The main technical challenge is to account for the spatial dependences introduced by rank deficiencies in the interference alignment schemes that typically rely on the independence of channel coefficients.
\end{abstract}

Index Terms-Channel capacity, degrees of freedom, interference channel, multiple-input multiple-output (MIMO), rank deficient channels, interference alignment

\section{INTRODUCTION}

$\mathbf{T}$ HE idea of interference alignment originated out of the studies of the index coding problem [1] and the degrees of freedom (DoF) of $X$ channels [2], [3] and $K$-user interference channels [4]. Over a relatively short period, it has found applications across a broad array of wireless and wired communication networks [5]. A variety of interference alignment schemes have enabled new and fundamental insights into the number of signal dimensions that are accessible in distributed communication networks where the output signals are linear functions of input signals (possibly with additive noise). However, the insights are mostly limited to the assumption of independent channel realizations. This is because with few exceptions, the studies of interference

Manuscript received October 15, 2013; revised July 26, 2014; accepted October 10, 2014. Date of publication November 20, 2014; date of current version December 22, 2014. This work was supported by the NSF under Award CCF-1161418 and Award CCF-1319104. This paper was presented at 2012 GLOBECOM.

The authors are with the Center of Pervasive Communications and Computing, Department of Electrical Engineering and Computer Science, University of California at Irvine, Irvine, CA 92697 USA (e-mail: srkrishn@uci.edu; abinesh.r@uci.edu; syed@uci.edu).

Communicated by A. Guillén i Fàbregas, Associate Editor for Communications.

Color versions of one or more of the figures in this paper are available online at http://ieeexplore.iee.org.

Digital Object Identifier 10.1109/TIT.2014.2372773 alignment schemes assume some form of independence (linear, probabilistic, algebraic, rational) of channel realizations. While channels are naturally independent across users in single-hop networks, dependencies often arise across antennas in MIMO interference networks. Understanding the impact of these dependencies on the signal dimensions of MIMO interference networks is important both from a practical and a theoretical perspective.

In this work we study spatial dependencies that are manifested as rank-deficiencies of the channel matrices in MIMO interference networks. Rank deficient channels are frequently encountered in MIMO networks, due to poor scattering, presence of single or very few paths, insufficient antenna-spacing and keyhole effects. While the implications of rank deficient channels are well understood for the single user point to point setting, much less is known for interference networks. In this work we study the degrees of freedom (DoF) of rank-deficient MIMO interference networks. To isolate the impact of spatial dependencies, we allow channels to vary independently across time and frequency. This also allows us to exploit the well-developed machinery of linear interference alignment schemes, which are appealing not only for their simplicity and robustness, but also because they tend to be DoF-optimal for time-varying channels.

Linear alignment schemes may be broadly classified into non-asymptotic and asymptotic schemes, based on whether the size of the linear precoding vector space required to approach the optimal DoF value is finite or infinite, respectively. Non-asymptotic schemes typically suffice for underconstrained systems, where the number of spatial dimensions (antennas) is sufficiently large relative to the number of alignment constraints (users). This is the case, e.g., in the 2-user and 3-user MIMO interference channels, studied in [6] and [7], respectively. Asymptotic schemes, based on a construction proposed by Cadambe and Jafar in [4] for the $K$-user interference channel with time-varying/frequencyselective channel coefficients (in short, the CJ scheme), are typically needed when the number of alignment constraints (users) dominates the number of spatial dimensions (antennas). In this work, we explore the implications of having rank deficient channel matrices for both asymptotic and nonasymptotic schemes.

\section{A. Background}

Our study of non-asymptotic schemes will focus on 2-user and 3-user MIMO interference channels, whereas asymptotic schemes will be studied through the $K$-user MIMO 
interference channel setting. The relevant background is summarized in this section.

1) Non-Asymptotic Schemes (2-User and 3-User MIMO Interference Channels): Under the assumption of independent channel realizations, i.e., full rank channels, the DoF of the 2-user MIMO interference channel with arbitrary number of antennas at each node are characterized in [6]. The DoF of the 3-user MIMO interference channel where all nodes have the same number of antennas are characterized by Cadambe and Jafar in [4]. The DoF of 3-user MIMO interference channel with $M$ antennas at each transmitter and $N$ antennas at each receiver are characterized in parallel works by Wang et al. in [7] and by Bresler et al. in [8]. While the achievability results are the same in the two works, the outer bounds presented by Wang et al. are strictly stronger. The outer bounds of Bresler et al. are restricted to the linear feasibility of [9] and [10] where only linear precoding schemes are considered, and precoding across multiple channel uses is not allowed. However, Wang et al. present information theoretic outer bounds that are also applicable to non-linear schemes, arbitrary channel extensions, and timevarying channels. Remarkably, inspite of the more general setting, the information theoretic bounds of [7] match the linear outer bounds of [8]. Since information theoretic bounds directly imply linear outer bounds, the linear outer bounds of Bresler et al. are immediately recovered as special cases of the information theoretic outer bounds of Wang et al. Comparing achievability and outer bounds, the two coincide in the sense of a spatially normalized DoF metric. Whether the achievability matches the outer bound without the spatial normalization is subject to the validity of the spatial invariance conjecture of Wang et al. [7], which essentially states that time, frequency and space dimensions are equivalent from a DoF perspective (so that there is no loss of generality in a spatial normalization). Remarkably, with the exception of the single-antenna setting, only non-asymptotic interference alignment schemes are used in [7], [8] to achieve the DoF outer bounds.

2) Asymptotic Schemes: For generic channels, the CJ scheme was introduced by Cadambe and Jafar in [4] to show that the $K$ user interference channel with a single antenna at each node (SISO setting) has a total of $\frac{K}{2}$ DoF almost surely. The key to this result was aligning interference almost perfectly in half of the received signal space by precoding over an asymptotically large number of channel uses, over independently time-varying or frequency-selective channels. The CJ scheme forms the basis of many, if not most, asymptotic schemes encountered in a variety of settings ranging from $X$ networks [11], cooperative and cognitive communications [12]-[14], to distributed storage exact repair [15] and multiple unicast network coding [16], and translates, quite remarkably, into the rational dimensions framework for constant channels as well [17], [18].

Originally proposed for the SISO setting, the CJ scheme was directly extended to the $K$ user MIMO interference channel in [4] by a decomposition approach, viewing a $K$ user interference channel where each node has $M$ antennas, as a $K M$ user interference channel where each node has a single antenna. The decomposition approach achieves the optimal DoF value of $\frac{K M}{2}$ for this network. Applying the CJ scheme to the SISO setting obtained by the decomposition of a MIMO interference channel, is also shown to be a DoF optimal strategy in [19] and [20] for $K$ user MIMO interference networks where each transmitter has $M$ antennas and each receiver has $N$ antennas, provided that the number of users exceeds a threshold that depends on $M, N$. The need for a SISO decomposition for the CJ scheme can be understood as follows: The CJ scheme requires commutativity of matrix multiplication, which is not satisfied by the generic channel matrices in MIMO networks (which produce non-commuting block diagonal channels). However, decomposing the MIMO network into a SISO network and allowing channel extensions over time/frequency creates diagonal channel matrices, which satisfy the commutative property. While at first, the limitation of having to decompose MIMO channels, may appear to be a limitation of the $\mathrm{CJ}$ scheme, it is remarkable that the CJ scheme remains DoF optimal in spite of the decomposition. The decomposability property is further discussed in [20]-[22].

3) Rank-Deficient MIMO Interference Networks: For rank deficient MIMO interference networks, much less is known. A study of achievable DoF is initiated by Chae et al. in [23] under the assumption that there are $M$ antennas at each transmitter, $N$ antennas at each receiver, and that the $N \times M$ channel matrix from each transmitter to each receiver is of rank $D$. However, in the absence of outer bounds for rankdeficient channels, the optimality of the achieved DoF is not settled. Following the preliminary version of this work [24], Zeng et al. have found the DoF for the 3-user rank deficient interference channel independently and in parallel work [25], with $M_{T}$ antennas at all transmitters and $M_{R}$ antennas at all receivers and with channel matrices of rank $D_{i}, i \in\{1,2,3\}$. At each receiver, the desired signal is assumed to arrive through a channel of rank $D_{0}$, interference from the 'previous' transmitter arrives through a channel of rank $D_{1}$ and the interference from the 'next' transmitter arrives through a channel of rank $D_{2}$. Other studies of rank-deficient wireless networks include the DoF characterization of 2-user rank deficient MIMO $X$ channel under arbitrary antenna configurations by Agustin and Vidal in [26].

\section{Summary OF CONTRIBUtions}

First, let us consider settings that correspond to non-asymptotic schemes. Interference alignment and zero-forcing through spatial beamforming are the core principles of non-asymptotic linear interference management schemes. Interestingly, rank-deficiencies impact the two in opposite ways, favoring one and limiting the other. Rankdeficiencies create more opportunity for zero-forcing because the channel null-space size is increased. However, there is less opportunity for interference alignment because reduced range spaces imply reduced overlaps between range spaces. Given the contrasting effects on alignment and zero-forcing, it is not clear a-priori whether the overall impact of rank-deficiencies should be positive or negative. Our results for rank-deficient 2-user and 3-user MIMO interference channels shed light on 
this tradeoff. For both 2-user and 3-user rank deficient MIMO interference channels, our focus is mainly on achievable schemes for constant channels, which can also be used for time-varying channels. For the 2-user rank deficient MIMO interference channel, we (i) provide a tight outer bound to show that the previously known achievable DoF found by Chae et al. in [23] in the symmetric case are optimal, and (ii) we generalize the result to fully asymmetric settings. For the 3-user rank deficient MIMO channel, we characterize the DoF of a cyclically symmetric setting where all nodes have the same number of antennas $(M)$. Our DoF results for the 3-user case are consistent with those derived in parallel work by Zeng et al. if we set $M_{T}=M_{R}$ in [25], however while the achievable scheme of [25] requires asymptotic number of symbol extensions when $M_{T}=M_{R}$, we present a non-asymptotic achievable scheme that requires at most two symbol extensions.

Next, let us consider asymptotic schemes, and in particular, the idea of decomposing the MIMO network into a SISO network where the CJ scheme is applicable. If the MIMO channels were comprised of independent channel coefficients, then the decomposition of the MIMO network into a SISO network preserves the channel independence requirements of the CJ scheme. With rank-deficiencies, however, this is no longer the case. The direct and cross channels are dependent in the decomposed SISO network and it is easy to see that the basic requirements of the $\mathrm{CJ}$ scheme are violated. If the CJ scheme is directly applied there must be a loss of DoF due to the channel dependencies. This observation is particularly ominous given that viable alternatives to the $\mathrm{CJ}$ scheme are not known for over-constrained interference networks. Our results for rank-deficient $K$-user MIMO interference channels shed light on this conundrum. For $K$-user rank deficient MIMO interference channel, we study achievable schemes for timevarying channels, which often serve as stepping stones to translate DoF results to constant channels. In particular, we show that for the $K$-user rank deficient interference channel, when all nodes have $M$ antennas, all direct channels have rank $D_{0}$, all cross channels are of rank $D$, and the channels are otherwise generic, the optimal DoF value per user is $\min \left(D_{0}, M-\frac{\min (M,(K-1) D)}{2}\right)$. Our result improves upon the best known achievable DoF from prior work, and we present a tight outer bound to prove its optimality.

Remarkably, our results indicate that for interference channels, the rank-deficiency of direct channels does not help and the rank-deficiency of cross-channels does not hurt. The main technical challenge in the paper is to account for the spatial dependencies introduced by rank deficiencies in the interference alignment schemes that typically rely on the independence of channel coefficients.

We start with a general system model which will be specialized in later sections for different settings.

\section{System MODEL}

The $K$-user MIMO interference channel is comprised of $K$ transmitters, $K$ receivers, and $K$ independent messages as shown in Fig. 1. Transmitter $k$, denoted

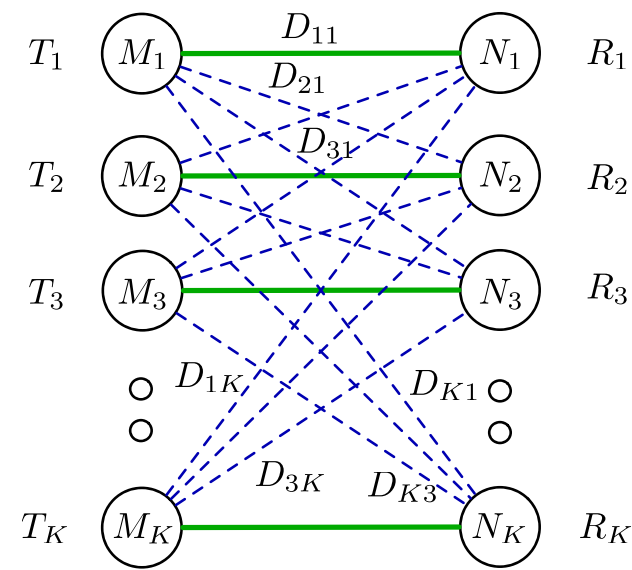

Fig. 1. $K$-user MIMO Interference Network with Rank Deficient Channels.

as $T_{k}$, is equipped with $M_{k}$ antennas and has message $W_{k}$ intended for its corresponding receiver, Receiver $k$, denoted as $R_{k}$, is equipped with $N_{k}$ antennas. At time index $t \in \mathbb{Z}^{+}$, Receiver $j$ observes the vector $Y_{j}(t) \in \mathbb{C}^{N_{j} \times 1}$ given by

$$
Y_{j}(t)=\sum_{i=1}^{K} \mathbf{H}_{j i}(t) X_{i}(t)+Z_{j}(t)
$$

wherein $X_{i}(t) \in \mathbb{C}^{M_{i} \times 1}$ is the vector sent from Transmitter $i$, $\mathbf{H}_{j i}(t) \in \mathbb{C}^{N_{j} \times M_{i}}$ is the channel matrix between Transmitter $i$ and Receiver $j$ and $Z_{j}(t) \in \mathbb{C}^{N_{j} \times 1}$ is the i.i.d. zero mean unit variance circularly symmetric complex additive white gaussian noise (AWGN) vector. Each transmitter must satisfy an average power constraint $\mathbb{E}\left(\left\|X_{i}(t)\right\|^{2}\right) \leq \rho$, where $\rho$ is referred to as the Signal-to-Noise Power Ratio, or the SNR. Global channel knowledge is assumed to be perfectly available at all nodes, and the transmitters are assumed to know the channels instantaneously.

The most important aspect of the system model for this work is the assumption that channels are rank-constrained, so that the channel matrix $\mathbf{H}_{j i}(t)$ has rank $D_{j i}$ almost surely. Aside from the rank-constraint, the channel matrices are generic, i.e., they possess no special structure. Mathematically, an $N_{j} \times M_{i}$ generic matrix subject to a rank-constraint $D_{j i}$ may be defined as the product of a pair of independently generated matrices of dimensions $N_{j} \times D_{j i}$ and $D_{j i} \times M_{i}$, each of which has its elements drawn from a continuous distribution with support bounded away from zero and infinity.

Achievable rates, capacity region, and $\mathrm{DoF}$ are defined in the standard sense (see [4]). In this work we are primarily interested in the sum-DoF value for almost all channel realizations, defined as $d_{\Sigma}=\lim _{\rho \rightarrow \infty} R_{\Sigma}(\rho) / \log (\rho)$, wherein $R_{\Sigma}(\rho)$ is the maximum sum rate of the channel at Signal-to-noise ratio, $\rho$. We also denote the sum DoF as $\operatorname{DoF}_{\Sigma}\left(M_{i}, N_{j}, D_{j i}\right)$, and the sum DoF normalized by the spatial dimension as

$$
\overline{\operatorname{DoF}}_{\Sigma}\left(M_{i}, N_{j}, D_{j i}\right)=\max _{q \in \mathbb{Z}_{+}} \frac{\operatorname{DoF}_{\Sigma}\left(q M_{i}, q N_{j}, q D_{j i}\right)}{q}
$$

Notation: $\mathbb{Z}_{+}$denotes the set of positive integers, and $\mathbb{C}$ denotes the set of complex numbers. For the matrix $\mathbf{H}$, 


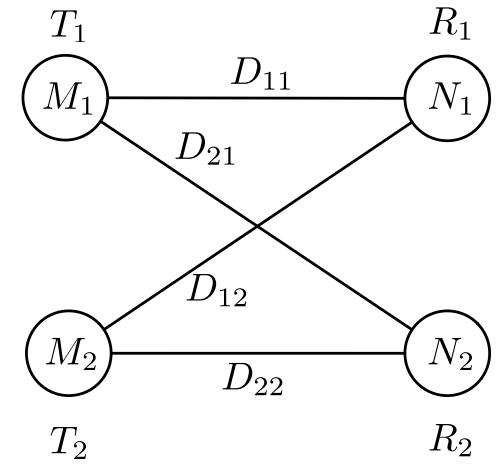

Fig. 2. 2-user Rank Deficient Interference Channel.

$\mathbf{H}(i,:)$ and $\mathbf{H}(:, j)$ denote its $i$ th row and $j$ th column vector, respectively. When dealing with $\mathbf{H}_{k(k+1)}$ and $\mathbf{H}_{k(k-1)}$, indexing is interpreted in a circular wrap-around manner, modulo the number of users, e.g., the $K$-th user is same as the 0 -th user. We use the notation $o(x)$ to represent any function $f(x)$ such that $\lim _{x \rightarrow \infty} \frac{f(x)}{x}=0$. We denote the number of columns of matrix $\mathbf{V}$ as $|\mathbf{V}|$, and $\mathbf{V}^{\dagger}$ is used to denote the conjugate transpose of matrix $\mathbf{V}$. The term nullspace refers to the right nullspace, unless otherwise explicitly mentioned. $(x)^{+}$indicates $\max (0, x) . \mathbf{I}_{M}$ denotes the $M \times M$ identity matrix and $\otimes$ denotes the Kronecker product. Matrices are notated using bold font while vectors are denoted with normal font.

\section{2-USER CHANNEL}

The DoF of the 2-user rank deficient interference channel is presented in the following theorem.

Theorem 1: For the 2-user rank deficient MIMO interference channel, the sum-DoF value is given by

$$
\operatorname{DoF}_{\Sigma}=\min \left\{D_{11}+D_{22}, M_{1}+N_{2}-D_{21}, M_{2}+N_{1}-D_{12}\right\}
$$

Placing the result in perspective with prior work, recall that in [23] Chae et al. have considered a symmetric version of the 2-user MIMO interference channel, for which they have established an achievable DoF value. Theorem 1 shows that the achievable DoF value of Chae et al. is optimal in the symmetric setting, and generalizes the result to arbitrary antenna configurations and arbitrary rank-constraints, shown in Figure 2. This DoF result holds for both time-varying and constant channel coefficients.

Note that the rank-deficiency of direct channels does not help the DoF and the rank-deficiency of cross-channels does not hurt. Since interference-alignment is not a possibility, the achievability is based on simple zero-forcing, which benefits from the increased null-space of cross channel matrices.

\section{A. Theorem 1: Proof of Achievability}

Since the proof is similar to that of the 2-user full rank interference channel [6], we do not repeat all the details. Transmitter $i$ has $M_{i}$ antennas, Receiver $j$ has $N_{j}$ antennas and the channel between Transmitter $i$ and Receiver $j$ is of rank $D_{j i}$. Figure 3 illustrates the proof setting with an example where $M_{1}=5, M_{2}=4, N_{1}=4, N_{2}=4, D_{11}=3, D_{22}=3$, $D_{12}=2$ and $D_{21}=4$, where a total of $5 \mathrm{DoF}$ are achieved.

Step 1: We consider a singular value decomposition (SVD) of the interference channels $\mathbf{H}_{12}=\mathbf{U}_{1} \Lambda_{12} \mathbf{V}_{1}^{\dagger}$ and $\mathbf{H}_{21}=\mathbf{U}_{2} \Lambda_{21} \mathbf{V}_{2}^{\dagger}$ wherein $\mathbf{U}_{1}, \mathbf{U}_{2}, \mathbf{V}_{1}, \mathbf{V}_{2}$ are $N_{1} \times N_{1}$, $N_{2} \times N_{2}, M_{2} \times M_{2}, M_{1} \times M_{1}$ unitary matrices, respectively. $\Lambda_{12}$ and $\Lambda_{21}$ are $N_{1} \times M_{2}, N_{2} \times M_{1}$ diagonal matrices with singular values of $\mathbf{H}_{12}, \mathbf{H}_{21}$ respectively on the main diagonal and zeros elsewhere. Using the standard MIMO SVD diagonalization approach as in [6], we absorb the unitary matrices into the corresponding input and output vectors as:

$$
\begin{aligned}
& \bar{Y}_{1}=\overline{\mathbf{H}}_{11} \bar{X}_{1}+\Lambda_{12} \bar{X}_{2}+\bar{Z}_{1} \\
& \bar{Y}_{2}=\overline{\mathbf{H}}_{22} \bar{X}_{2}+\Lambda_{21} \bar{X}_{1}+\bar{Z}_{2}
\end{aligned}
$$

where $\bar{Y}_{1}=\mathbf{U}_{1}^{\dagger} Y_{1}, \bar{Y}_{2}=\mathbf{U}_{2}^{\dagger} Y_{2}, \bar{X}_{1}=\mathbf{V}_{2}^{\dagger} X_{1}, \bar{X}_{2}=$ $\mathbf{V}_{1}^{\dagger} X_{2}, \bar{Z}_{1}=\mathbf{U}_{1}^{\dagger} Z_{1}, \bar{Z}_{2}=\mathbf{U}_{2}^{\dagger} Z_{2}, \overline{\mathbf{H}}_{11}=\mathbf{U}_{1}^{\dagger} \mathbf{H}_{11} \mathbf{V}_{2}$ and $\overline{\mathbf{H}}_{22}=\mathbf{U}_{2}^{\dagger} \mathbf{H}_{22} \mathbf{V}_{1}$. Here, $\bar{Y}_{j}, \bar{Z}_{j}, \forall j \in\{1,2\}$ are $N_{j} \times 1$ vectors and $\bar{X}_{i}, \forall i \in\{1,2\}$ are $M_{i} \times 1$ vectors. Element $m$ ( $m$-th row) of $\bar{X}_{i}, \bar{Y}_{i}$ are represented as $\bar{X}_{i}^{m}, \bar{Y}_{i}^{m}$, respectively. Since first $D_{12}$ columns of $\Lambda_{12}$ have nonzero values on the diagonal and other columns are zeros, only $\bar{X}_{2}^{1}, \bar{X}_{2}^{2}, \ldots, \bar{X}_{2}^{D_{12}}$ present interference from $T_{2}$ at $R_{1}$. Similarly only $\bar{X}_{1}^{1}, \bar{X}_{1}^{2}, \ldots, \bar{X}_{1}^{D_{21}}$ present interference from $T_{1}$ at $R_{2}$. Thick lines in Figure 3 represent interference links after diagonalization, and there are 2 parallel paths from $T_{2}$ to $R_{1}$ and 4 parallel paths from $T_{1}$ to $R_{2}$.

Step 2: At Transmitter $T_{1}$, inputs $\bar{X}_{1}^{1}, \bar{X}_{1}^{2}, \ldots, \bar{X}_{1}^{\left(M_{1}-D_{11}\right)}$ are set to zero, i.e., we do not transmit on these inputs, denoted as $M_{1}^{\prime}=M_{1}-D_{11}$. This leaves $D_{11}$ available inputs, $\bar{X}_{1}^{\left(M_{1}-D_{11}+1\right)}, \ldots, \bar{X}_{1}^{M_{1}}$ at $T_{1}$. In Figure 3, 2 transmit antennas have inputs set to zero (white circles) and remaining 3 dark circles indicate the available inputs at $T_{1}$.

Step 3: At Receiver $R_{1}, D_{11}=3$ is the dimension of desired signal received from $T_{1}$. Hence we consider only outputs $\bar{Y}_{1}^{1}, \bar{Y}_{1}^{2}, \ldots, \bar{Y}_{1}^{D_{11}}$ and discard remaining outputs $\bar{Y}_{1}^{\left(D_{11}+1\right)}, \ldots, \bar{Y}_{1}^{N_{1}}$ marked in white circles. Receiver $R_{1}$ uses $D_{11}$ dimensions for its desired signal since its desired channel rank is $D_{11}$. Hence $N_{1}^{\prime}=N_{1}-D_{11}$ is the number of tolerable interference dimensions at $R_{1}$. Receiver $R_{1}$ is exposed to $D_{12}$ dimensions from the $M_{2}$ dimensional space available to Transmitter $T_{2}$ since the channel between $T_{2}$ and $R_{1}$ is of rank $D_{12}$. Since $R_{1}$ can tolerate only $N_{1}^{\prime}$ dimensions of interference, $T_{2}$ uses only $N_{1}^{\prime}$ of these $D_{12}$ dimensions, transmitting nothing on (zero forcing) the remaining $D_{12}-N_{1}^{\prime}$ dimensions. In addition, $T_{2}$ is free to transmit on the $M_{2}-D_{12}$ dimensions that are not seen by $R_{1}$. Thus, $T_{2}$ transmits its message using $M_{2}-D_{12}+N_{1}^{\prime}=M_{2}-\left(D_{11}+D_{12}-N_{1}\right)$ dimensions. Figure 3 illustrates such an example.

Step 4: Discarding $\left(D_{12}+D_{11}-N_{1}\right)$ inputs at $T_{2}$ ensures that at Receiver $R_{1}$, interference is eliminated and $R_{1}$ can decode the message from Transmitter $T_{1}$ to achieve $D_{11}$ DoF.

Step 5: Receiver $R_{2}$ receives interference from Transmitter $T_{1}$ over channel of rank $D_{21}$. In Step 2, $M_{1}^{\prime}$ inputs have been set to zero, hence remaining $\left(D_{21}-M_{1}^{\prime}\right)^{+}$inputs cause interference at $R_{2}$. In order to eliminate interference from $T_{1}$, Receiver $R_{2}$ discards $\left(D_{21}-M_{1}^{\prime}\right)^{+}$outputs. Therefore, $R_{2}$ receives signal from $T_{2}$ only on its $N_{2}-\left(D_{21}-M_{1}^{\prime}\right)^{+}$ 


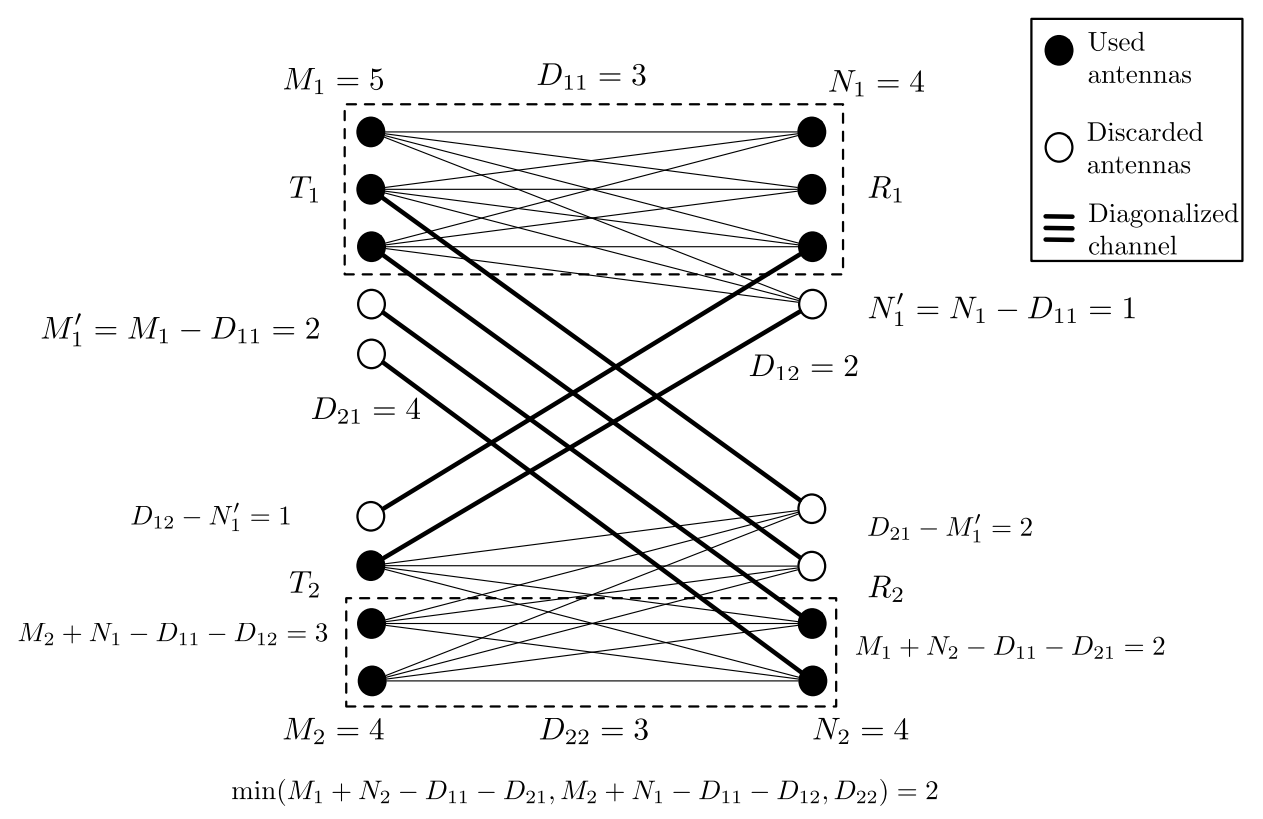

Fig. 3. Achievability for 2-user Rank deficient channel.

remaining outputs. In Figure 3, Transmitter $T_{1}$ sets $M_{1}^{\prime}=2$ of its inputs to zero, and Receiver $R_{2}$ discards $\left(D_{21}-M_{1}^{\prime}\right)^{+}=2$ outputs. $R_{2}$ decodes its signal using remaining $N_{2}-\left(D_{21}-\right.$ $\left.M_{1}^{\prime}\right)^{+}=2$ outputs.

Step 6: From step 3, we have $M_{2}-\left(D_{11}+D_{12}-N_{1}\right)$ inputs available at $T_{2}$ so that no interference is caused at $R_{1}$. From step 5 , we have $N_{2}-\left(D_{11}+D_{21}-M_{1}\right)^{+}$outputs available at $R_{2}$ that are interference-free. Channel between $T_{2}$ and $R_{2}$ is of rank $D_{22}$. Hence communication between $T_{2}$ and $R_{2}$ takes place with DoF of $\min \left(M_{2}-\left(D_{11}+D_{12}-N_{1}\right), N_{2}-\left(D_{11}+\right.\right.$ $\left.\left.D_{21}-M_{1}\right)^{+}, D_{22}\right)$.

Combining Steps 4 and 6, we have established achievability of $D_{11}+\min \left(M_{2}-\left(D_{11}+D_{12}-N_{1}\right), N_{2}-\left(D_{11}+D_{21}-\right.\right.$ $\left.M_{1}\right)^{+}, D_{22}$ ) total DoF for 2-user channel. This expression can be evaluated to be equal to $\min \left\{D_{11}+D_{22}, M_{1}+N_{2}-\right.$ $\left.D_{21}, M_{2}+N_{1}-D_{12}\right\}$. Setting inputs or outputs to zero is equivalent to performing zero-forcing at the transmitter or receiver, respectively.

\section{B. Theorem 1: Proof of Outer Bound}

The trivial outer bound on total DoF of $D_{11}+D_{22}$ is obvious for this channel. The following converse proof is similar to that of full rank channels (refer [6, Th. 1]), and so, once again we only present a proof sketch for rank-deficient channels.

For the sum capacity of this channel to be bounded above by 2 constituent MAC channels, each receiver must be able to decode messages from both transmitters. For this, the receiver must have access to the full interference signal space, i.e., it does not get zero-forced at the transmitters. Similar to [6, Th. 1], we replace the original additive noise at one receiver, say $R_{1}$, with noise having different (less power) statistics. Note that this does not make the capacity region smaller since the original noise statistics can be obtained by locally generating and adding noise at $R_{1}$. Hence, if $R_{1}$ was originally able to decode its intended message, it is still capable of decoding its message with modified noise statistics. In this sense, noise is modified at Receiver $R_{1}$, if needed, so that it sees a better channel than Receiver $R_{2}$, and the message intended for Receiver $R_{2}$ becomes decodable at Receiver $R_{1}$.

In the 2-user rank deficient MIMO interference channel, Receiver $R_{1}$ can access only a $D_{12}$ dimensional signal space of Transmitter $T_{2}$ in its $M_{2}$ dimensional space. This implies, $T_{2}$ can zero-force part of its signal to $R_{1}$ and $R_{1}$ cannot decode the message from $T_{2}$ by reducing noise. Hence only through additional antennas at $R_{1}$ can it access the full signal space of $T_{2}$. Additional receiver antennas cannot hurt, so the converse argument is not violated. To this end, we add $M_{2}-D_{12}$ antennas at $R_{1}$. The channel coefficients corresponding to the new antennas are generic, so that the interference channel between $T_{2}$ and $R_{1}$, now a matrix of size $\left(N_{1}+M_{2}-D_{12}\right) \times M_{2}$, will have full rank almost surely. With this, $R_{1}$ can obtain a stronger channel to input of $T_{2}$, so that if $R_{2}$ can decode the message of $T_{2}$, so can $R_{1} . R_{1}$ can locally generate noise and add to its received signal which is statistically equivalent noise signal as seen by $R_{2}$. After decoding and subtracting its desired signal from $T_{1}, R_{1}$ has a less noisy channel to $T_{2}$ and can decode the message sent by $T_{2}$. Similarly, additional antennas are added at Receiver $R_{2}$, so that it can access the full signal space of Transmitter $T_{1}$. The interference channel between $T_{1}$ and $R_{2}$, a matrix of size $\left(N_{2}+M_{1}-D_{21}\right) \times M_{1}$, has full rank almost surely. With this, $R_{2}$ can obtain a stronger channel to input of $T_{1}$, so that if $R_{1}$ can decode the message of $T_{1}$, so can $R_{2}$.

Now, we argue that the sum capacity is bounded above by corresponding MAC channels $\left(M_{1}, M_{2}, N_{1}+M_{2}-D_{12}\right)$ and $\left(M_{1}, M_{2}, N_{2}+M_{1}-D_{21}\right)$ with modified additive noise. Since $\left(N_{2}+M_{1}-D_{21}\right) \geq M_{1}$ and $\left(N_{1}+M_{2}-D_{12}\right) \geq M_{2}$, it can be seen that [6, Th. 1] holds true for above argument with $N_{1}$ modified as $N_{1}+M_{2}-D_{12}$ and $N_{2}$ modified as 
$N_{2}+M_{1}-D_{21} . R_{1}$ can decode its message and subtract from its received signal vector, and we assume a genie provides $X_{1}$ to $R_{2}$, so that $R_{2}$ can subtract out interference from $T_{1}$. While initial output vectors $Y_{1}$ and $Y_{2}$ are of size $\left(N_{1}+M_{2}-D_{12}\right) \times 1$ and $\left(N_{2}+M_{1}-D_{21}\right) \times 1$ respectively, after noise reduction and SVD operations, output vectors $Y_{1 \text { new }}$ and $Y_{2 \text { new }}$ are both of size $M_{2} \times 1$. With these changes, $R_{1}$ and $R_{2}$ would be able to decode both messages. Hence, the total DoF value is upperbounded as $\mathrm{DoF} \leq \min \left(D_{11}+D_{22}, N_{2}+M_{1}-D_{21}, N_{1}+\right.$ $\left.M_{2}-D_{12}\right)$. Thus, we get the converse result of Theorem 1 .

Remark 1: Reciprocity holds true for rank deficient channels similar to full rank channels, i.e., DoF is unaffected if $M_{1}$ and $M_{2}$ are switched with $N_{1}$ and $N_{2}$ respectively.

Remark 2: For the symmetric special case, i.e., the $(M, N, D)$ MIMO interference channel where each transmitter has $M$ antennas, each receiver has $N$ antennas and all channel matrices are of rank $D$, optimal DoF can be calculated as $\min (M+N-D, 2 D)$, which is the same as the achievable DoF value established by Chae et al. [23], now proved to be optimal.

\section{3-USER CHANNEL}

To avoid an explosion of parameters when considering more than 2 users, we impose certain assumptions of symmetry. For the 3-user rank deficient interference channel, we assume that all transmitters and receivers have $M$ antennas, and channels $\mathbf{H}_{(j+k) j}$ are of rank $D_{k}, k \in\{0,1,2\}$. Thus, at each receiver, the desired signal arrives through a channel of rank $D_{0}$, interference from the 'previous' transmitter arrives through a channel of rank $D_{1}$ and the interference from the 'next' transmitter arrives through a channel of rank $D_{2}$, where transmitter and receiver indices are circularly wrapped around modulo 3. Under this assumption of symmetry, the DoF result is presented in the following theorem.

Theorem 2: For the 3-user rank deficient MIMO interference channel with $M$ antennas at each node, and channels $H_{(j+k) j}$ restricted to rank $D_{k}, j, k \in\{0,1,2\}$, the spatially normalized DoF value per user is given by

$$
\frac{\overline{\mathrm{DoF}}_{\Sigma}}{3}=\min \left\{D_{0}, M-\frac{\min \left(M, D_{1}+D_{2}\right)}{2}\right\}
$$

Placing the result into perspective, we note that the DoF value in Theorem 2 represents a strict improvement over the achievable DoF previously established by Chae et al. in [23], and matches the achievable DoF value established in parallel work by Zeng et al. in [25]. Although the results are consistent with those of [25], our achievable scheme requires atmost 2 symbol extensions while [25] involves large number of symbol extensions for the symmetric $M_{T}=M_{R}$ case. We also present a tight information theoretic outer bound that establishes the optimality of this DoF value.

The spatially normalized DoF result holds for both time-varying and constant channel coefficients. This follows similar to [7, Th. 1], by scaling the number of antennas by $q=2$, when DoF is non-integer. For channel with time-varying coefficients, Theorem 2 is also the DoF value, achievable with symbol extensions. Based on the spatial-scale invariance property [7], which is consistent for a wide variety

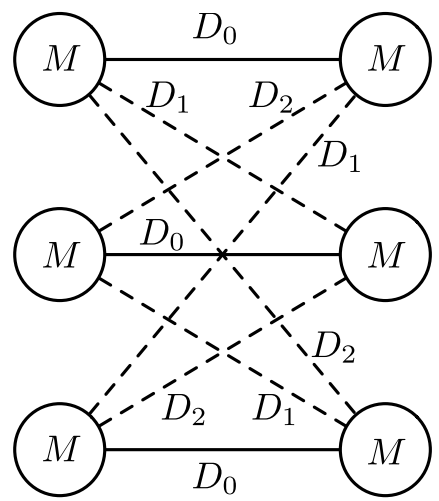

Fig. 4. 3-user Rank Deficient Interference Channel.

of networks, we conjecture that the result is also the DoF for the 3-user rank-deficient channel with constant coefficients.

The result of Theorem 2 is consistent with the observation that the rank-deficiency of cross-channels does not hurt and the rank-deficiency of direct channels does not help. Since the rank-deficiency of cross-channels increases opportunities for zero-forcing and reduces the opportunities for interference alignment, it is evident that the gain from increased zero-forcing more than offsets the loss from reduced interference alignment. Compared to the full-rank case where everyone achieves half the cake, it is remarkable that halfthe-cake (i.e., $M / 2$ DoF per user) remains achievable as long as the direct channels support it.

We consider the setting shown in Figure 4.

\section{A. Theorem 2: Proof of Achievability}

The achievability proof for the 3-user rank deficient interference channel is first presented for cases where direct channels are full rank. Later, achievability with rank deficient direct channels is discussed. We categorize beamforming vectors used at Transmitter $k$ to 4 types:

$\mathbf{V}_{k}^{Z a}$ - Zero-forcing vectors in the nullspace of $\mathbf{H}_{(k-1) k}$, maximum number of linearly independent vectors chosen can be $\min \left(D_{1}, M-D_{2}\right)$ (independent from $\left.\mathbf{V}_{k}^{Z c}\right)$. Vectors used at Transmitter $k$ will not cause interference at Receiver $k-1$.

$\mathbf{V}_{k}^{Z b}$ - Zero-forcing vectors in the nullspace of $\mathbf{H}_{(k+1) k}$, maximum number of linearly independent vectors chosen can be $\min \left(D_{2}, M-D_{1}\right)$ (independent from $\left.\mathbf{V}_{k}^{Z c}\right)$. Vectors used at Transmitter $k$ will not cause interference at Receiver $k+1$.

$\mathbf{V}_{k}^{Z c}$ - Zero-forcing vectors in the common nullspace of $\mathbf{H}_{(k-1) k}$ and $\mathbf{H}_{(k+1) k}$ (overlapping dimensions in the 2 nullspaces). Maximum number of linearly independent vectors chosen can be $M-D_{1}-D_{2}$ since $M-D_{1}$ and $M-D_{2}$ dimensional generic nullspaces overlap in a $M-D_{1}-D_{2}$ dimensional space at each transmitter (when $M \geq D_{1}+D_{2}$ ). Vectors chosen in these overlapping dimensions do not cause interference at either of the 2 unintended receivers.

$\mathbf{V}_{k}^{A}$ - Alignment vectors that align signal at a receiver in the span of interference from other unintended transmitter. Maximum number of linearly independent vectors chosen can be $D_{1}+D_{2}-M$ since $D_{1}$ and $D_{2}$ dimensional generic interference subspaces overlap in $D_{1}+D_{2}-M$ dimensional space at each receiver (when $M<D_{1}+D_{2}$ ). 
TABLE I

Achievable Dof in 3-User Channel for Different $D_{1}, D_{2}$ With $D_{0}=M$

\begin{tabular}{|l|l|l|l|l|l|l|l|}
\hline Case & $\mathbf{D}_{\mathbf{1}}+\mathbf{D}_{\mathbf{2}}$ & $\left|\mathbf{V}_{k}^{Z a}\right|+\left|\mathbf{V}_{k}^{Z b}\right|$ & $\left|\mathbf{V}_{k}^{Z c}\right|$ & $\left|\mathbf{V}_{k}^{A}\right|$ & $\operatorname{dim}($ Int $)$ & $\operatorname{dim}($ Des $)$ & Total \\
\hline 1 & $0<D_{1}+D_{2} \leq M$ & $\frac{D_{1}+D_{2}}{2}$ & $M-\left(D_{1}+D_{2}\right)$ & 0 & $\frac{D_{1}+D_{2}}{2}$ & $M-\frac{D_{1}+D_{2}}{2}$ & $M$ \\
\hline 2 & $M<D_{1}+D_{2} \leq \frac{3 M}{2}$ & $\frac{M}{2}$ & 0 & 0 & $\frac{M}{2}$ & $\frac{M}{2}$ & $M$ \\
\hline 3 & $\frac{3 M}{2}<D_{1}+D_{2} \leq 2 M$ & $2 M-D_{1}-D_{2}$ & 0 & $D_{1}+D_{2}-\frac{3 M}{2}$ & $\frac{M}{2}$ & $\frac{M}{2}$ & $M$ \\
\hline
\end{tabular}

Different cardinalities are chosen for these 4 types of beamforming vectors to form the transmit beamforming matrix. The beamforming matrix at each transmitter is then of the form $\mathbf{V}_{k}=\left[\begin{array}{llll}\mathbf{V}_{k}^{Z a} & \mathbf{V}_{k}^{Z b} & \mathbf{V}_{k}^{Z c} & \mathbf{V}_{k}^{A}\end{array}\right]$. We now discuss achievability by analyzing the beamforming vector cardinalities listed in Table I and by using linear dimension counting arguments.

Using Table I, we first analyze the setting in which direct channels are full rank and cross channels are rank deficient. The first 2 cases correspond to zero-forcing based achievability schemes, and the last case involves interference alignment.

For convenience, only the sum cardinality of the chosen zero-forcing vectors $\mathbf{V}_{k}^{Z a}$ and $\mathbf{V}_{k}^{Z b}$ is specified, i.e., $\left|\mathbf{V}_{k}^{Z a}\right|+\left|\mathbf{V}_{k}^{Z b}\right|$. This is because each of these vectors chosen at a transmitter helps in cancelling interference at one receiver but causes interference at another receiver. Since we have 2 unintended transmitters causing interference, these zero-forcing vectors can be treated in the same manner. $\operatorname{dim}$ (Desired) and $\operatorname{dim}$ (Interference) are the number of desired and interference signal dimensions seen at each receiver respectively. Then we have,

$$
\begin{aligned}
\operatorname{dim}(\text { Desired }) & =\left|\mathbf{V}_{k}^{Z a}\right|+\left|\mathbf{V}_{k}^{Z b}\right|+\left|\mathbf{V}_{k}^{Z c}\right|+\left|\mathbf{V}_{k}^{A}\right| \\
\operatorname{dim}(\text { Interference }) & =\left|\mathbf{V}_{k}^{Z a}\right|+\left|\mathbf{V}_{k}^{Z b}\right|+\left|\mathbf{V}_{k}^{A}\right|
\end{aligned}
$$

While the first relation is trivial, the second one can be explained as follows: $\mathbf{V}_{k}^{Z c}$ at Transmitter $k$ do not cause interference at both unintended receivers. Therefore $\operatorname{dim}$ (Interference) does not contain that term. Further, both zero-forcing (using non-overlapping nullspace) and interference alignment are similar in the sense that, a vector chosen for zero-forcing one receiver causes interference at the other receiver, and a vector chosen for aligning interference at one receiver causes interference at another. Hence at each receiver, $\operatorname{dim}$ (Interference) is the sum of the number of zero-forcing vectors (using non-overlapping nullspace) and the number of Interference alignment vectors. Overlapping nullspaces and interference are illustrated in Fig 5.

For the first case of Table I, $\left|\mathbf{V}_{k}^{A}\right|=0$ since interference alignment is not possible $\left(D_{1}+D_{2} \leq M\right)$. $\left|\mathbf{V}_{k}^{Z c}\right|$ is chosen to be the maximum possible overlapping nullspace dimensions. Remaining vectors are chosen from the nonoverlapping nullspace and chosen number of vectors $\left|\mathbf{V}_{k}^{Z a}\right|+$ $\left|\mathbf{V}_{k}^{Z b}\right|<D_{1}+D_{2}$, maximum number of non-overlapping nullspace dimensions. At each receiver, interference occupies $\left|\mathbf{V}_{k}^{Z a}\right|+\left|\mathbf{V}_{k}^{Z b}\right|$ dimensions.

For the second and third cases, $\left|\mathbf{V}_{k}^{Z c}\right|=0$ since there are no overlapping nullspace dimensions at the transmitters $\left(D_{1}+D_{2}>M\right)$. For case 2, though alignment is possible, beamforming matrix can be formed with the zero-forcing vectors only, i.e., $\left|\mathbf{V}_{k}^{Z a}\right|+\left|\mathbf{V}_{k}^{Z b}\right|$ can be chosen as $\frac{M}{2}$. This is because $\frac{M}{2} \leq 2 M-D_{1}-D_{2}$, dimensions in the nullspaces of $\mathbf{H}_{(k-1) k}$ and $\mathbf{H}_{(k+1) k}$.

Case 3 involves both zero forcing and interference alignment. At Transmitter $k \in\{1,2,3\}, M-D_{1}$ symbols are sent along the $M-D_{1}$ dimensional null space of the channel to Receiver $k-1$ and $M-D_{2}$ symbols are sent along the $M-D_{2}$ dimensional null space of the channel to Receiver $k+1$. This is performed by choosing columns of a full rank linear transformation $\mathbf{T}_{k}$ to be beamforming vectors $\mathbf{V}_{k}^{Z a}$ of size $M-D_{1}$ and $\mathbf{V}_{k}^{Z b}$ of size $M-D_{2}$.

$$
\mathbf{H}_{(k-1) k} \mathbf{V}_{k}^{Z a}=0, \quad \mathbf{H}_{(k+1) k} \mathbf{V}_{k}^{Z b}=0 \quad k \in\{1,2,3\}
$$

The remaining $D_{1}+D_{2}-M$ dimensional space at the transmitter will be used to send the remaining $M / 2-\left(M-D_{1}\right)-\left(M-D_{2}\right)=D_{1}+D_{2}-3 M / 2$ symbols that participate in interference alignment. Since the cross channel matrices are rank-deficient, eigen vector solution of [4] cannnot be used directly, since inverse of the matrices do not exist. Hence, one of the challenging aspects is to align vectors using rank deficient channel matrices. We will now show that using appropriate linear transformations at the transmitters and the receivers, interference alignment can be performed.

Within the $M$ dimensions available to Receiver $k$, the $D_{1}$ dimensional signal space accessible from Transmitter $k-1$ overlaps with the $D_{2}$ dimensional signal space accessible from Transmitter $k+1$ in a $\left(D_{1}+D_{2}-M\right)$ dimensional subspace. If these overlapping spaces can be accessed at all the transmitters and the receivers, interference alignment can be performed. Note that at the transmitters, we consider both zero-forcing and alignment vectors to access the $D_{1}$ or $D_{2}$ dimensional subspaces.

At the 3 receivers, matrices $\hat{\mathbf{R}}_{k}, k \in\{1,2,3\}$ of size $M \times\left(D_{1}+D_{2}-M\right)$ are constructed, that represent the signal space overlap of $D_{1}$ or $D_{2}$ dimensional subspaces seen from Transmitter $k-1$ and $k+1$ respectively.

$$
\hat{\mathbf{R}}_{k}=\operatorname{span}\left(\mathbf{H}_{k(k-1)}\right) \cap \operatorname{span}\left(\mathbf{H}_{k(k+1)}\right) \quad k \in\{1,2,3\}
$$

wherein $A \cap B$ denotes the intersection of $A$ and $B$, which can be identified as $\mathcal{N}(\mathcal{N}(A) \cup \mathcal{N}(B))$, and $\mathcal{N}(X)$ denotes the nullspace of $X$.

Interference will be aligned in these receiver signal spaces $\hat{\mathbf{R}}_{k}$. These overlapping signal spaces $\hat{\mathbf{R}}_{k}$, are projected back to the transmitters, such that the following equations are satisfied.

$$
\begin{aligned}
& \mathbf{H}_{(k+1) k} \tilde{\mathbf{T}}_{k}^{1}=\hat{\mathbf{R}}_{k+1} \\
& \mathbf{H}_{(k-1) k} \tilde{\mathbf{T}}_{k}^{2}=\hat{\mathbf{R}}_{k-1} \quad k \in\{1,2,3\}
\end{aligned}
$$




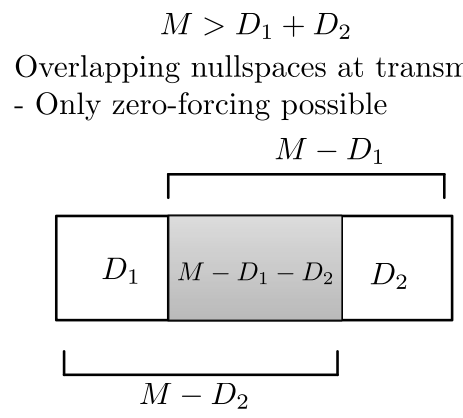

Fig. 5. $M$-dimensional signal space in 3-user interference channel.

wherein the matrices $\tilde{\mathbf{T}}_{k}^{1}, \tilde{\mathbf{T}}_{k}^{2}$ are of size $M \times\left(D_{1}+D_{2}-M\right)$. The matrices $\tilde{\mathbf{T}}_{k}^{1}, \tilde{\mathbf{T}}_{k}^{2}$ do not include vectors from the nullspaces of channels $\mathbf{H}_{(k+1) k}$ and $\mathbf{H}_{(k-1) k}$, respectively. After projecting back the signal spaces, they are combined with zero-forcing vectors to identify the signal space seen from Transmitter $k$ at the receivers $k-1$ and $k+1$. With this, Transmitter $k$ has $D_{1}$ dimensional space seen at Receiver $k+1$ and $D_{2}$ dimensional space seen at Receiver $k-1$, which overlap in a $\left(D_{1}+D_{2}-M\right)$ dimensional space, denoted as $\hat{\mathbf{T}}_{k}, k \in\{1,2,3\}$.

$$
\hat{\mathbf{T}}_{k}=\operatorname{span}\left(\left[\begin{array}{ll}
\tilde{\mathbf{T}}_{k}^{1} & \mathbf{V}_{k}^{Z b}
\end{array}\right]\right) \cap \operatorname{span}\left(\left[\begin{array}{ll}
\tilde{\mathbf{T}}_{k}^{2} & \mathbf{V}_{k}^{Z a}
\end{array}\right]\right)
$$

In order to align interference, above $M \times\left(D_{1}+D_{2}-M\right)$ submatrix, $\hat{\mathbf{T}}_{k}$ will be constructed, such that the same space is seen at both unintended receivers $k-1$ and $k+1$. Linear transformations $\hat{\mathbf{T}}_{k}, \hat{\mathbf{R}}_{k}$ represent the signal space overlap at the transmitters and the receivers, identification of which enables us to perform one-one alignment of vectors, as follows.

Transmitter $k$ uses the following $M \times M$ linear transformation $\mathbf{T}_{k}$ using the signal space overlap matrix, $\hat{\mathbf{T}}_{k}$ and zero-forcing vectors.

$$
\mathbf{T}_{k}=\left[\begin{array}{lll}
\mathbf{V}_{k}^{Z a} & \hat{\mathbf{T}}_{k} & \mathbf{V}_{k}^{Z b}
\end{array}\right] \quad k \in\{1,2,3\}
$$

Receiver $k$ sees $M-D_{1}$ dimensional interference from Transmitter $k-1$ and $M-D_{2}$ dimensional interference from Transmitter $k+1$. These $\left(M-D_{1}\right)+\left(M-D_{2}\right)$ interference symbols are zero-forced by projecting the $M$ dimensional received space into the $M-\left(M-D_{1}\right)-\left(M-D_{2}\right)$ dimensional space that is orthogonal to the interference symbols. This is performed using a full rank linear transformation $\mathbf{R}_{k}$ of size $\left(D_{1}+D_{2}-M\right) \times M$ at Receiver $k$.

$$
\mathbf{R}_{k}\left[\mathbf{H}_{k(k-1)} \mathbf{V}_{k-1}^{Z a} \quad \mathbf{H}_{k(k+1)} \mathbf{V}_{k+1}^{Z b}\right]=0, \quad k \in\{1,2,3\}
$$

With this, residual interference at Receiver $k$ due to zeroforcing beamforming vectors chosen at all transmitters are zero-forced. For the remaining symbols, i.e., for the remaining interference alignment problem, the zero forcing operations at the transmitters and receivers, described thus far leave us with a 3-user MIMO interference channel with $D_{1}+D_{2}-M$ input dimensions at each transmitter and $D_{1}+D_{2}-M$ dimensions at each receiver, with the following channel matrices. This is illustrated in Figure 6.

$$
\overline{\mathbf{H}}_{k j}=\mathbf{R}_{k} \mathbf{H}_{k j} \mathbf{T}_{j}
$$

We have constructed $\overline{\mathbf{H}}^{\prime}{ }_{j i}$ by considering $D_{1}+D_{2}-M$ columns of matrix $\overline{\mathbf{H}}_{j i}$ after excluding first $M-D_{1}$ and last $M-D_{2}$ columns. Since $D_{1}+D_{2}-M$ is not larger than $D_{1}, D_{2}$, these channels are full rank, generic channels over which the eigenvectors-based interference alignment solution of [4] can be directly applied to send the remaining $D_{1}+D_{2}-3 M / 2$ symbols (Note that 2 channel uses are needed for the aligned symbols if $M$ is an odd number, each corresponding to a new set of zero-forcing symbols). Thus, the effective receiver sees a $D_{1}+D_{2}-M$ dimensional generic space within which $D_{1}+$ $D_{2}-3 M / 2$ aligned interference dimensions and $\left(M-D_{1}\right)+$ $\left(M-D_{2}\right)+\left(D_{1}+D_{2}-3 M / 2\right)$ desired dimensions are resolved.

The beamforming matrices $\overline{\mathbf{V}}_{k}$ have $\left(M-D_{1}\right)+\left(M-D_{2}\right)$ columns of the identity matrix, shown on the leftmost and the rightmost column in the example below. Remaining columns of $\overline{\mathbf{V}}_{k}$ are based on the eigen-vector solution of dimension $D_{1}+D_{2}-M$ and rows of zeros above and below. Suppose $M=6$ and $D_{1}=D_{2}=5, \overline{\mathbf{V}}_{k}$ constructed with 2 zero-forcing vectors and 1 alignment vector, have the following structure.

$$
\overline{\mathbf{V}}_{k}=\left[\begin{array}{ccc}
1 & 0 & 0 \\
0 & v_{k 1}^{a} & 0 \\
0 & v_{k 2}^{a} & 0 \\
0 & v_{k 3}^{a} & 0 \\
0 & v_{k 4}^{a} & 0 \\
0 & 0 & 1
\end{array}\right]
$$

wherein $\overline{\mathbf{V}}_{k}^{A}=\left[\begin{array}{llll}v_{k 1}^{a} & v_{k 2}^{a} & v_{k 3}^{a} & v_{k 4}^{a}\end{array}\right]^{T}$ is the interference alignment vector constructed as in [4], which is then extended with $M-D_{1}$ rows of zeros above and $M-D_{2}$ rows of zeros below to form $\mathbf{V}_{k}^{A}$. Due to the construction of $\mathbf{T}_{k}$ using the signal space overlap $\hat{\boldsymbol{T}}_{k}$, vectors $\overline{\boldsymbol{V}}_{k}^{A}$ align one-one at the receivers. The resultant beamforming matrix $\mathbf{V}_{k}$ used at Transmitter $k$ is then

$$
\mathbf{V}_{k}=\mathbf{T}_{k} \overline{\mathbf{V}}_{k}=\left[\begin{array}{lll}
\mathbf{V}_{k}^{Z a} & \mathbf{V}_{k}^{A} & \mathbf{V}_{k}^{Z b}
\end{array}\right]
$$

Linear transformations at all transmitters and receivers $\mathbf{T}_{k}, \mathbf{R}_{k}$ are full rank matrices based on construction described. It can be noted that matrices $\overline{\mathbf{V}}_{k}$ and $\mathbf{V}_{k}$ are full rank since columns are linearly independent due to orthogonal construction of $\overline{\mathbf{V}}_{k}$. Note that desired channels are not used in the design of precoding vectors, which maintains their generic character and thereby the linear independence of desired signal vectors from the interference. We also note that a similar layered precoding approach is presented in [25] as well. 
Channel - $H_{j i}$

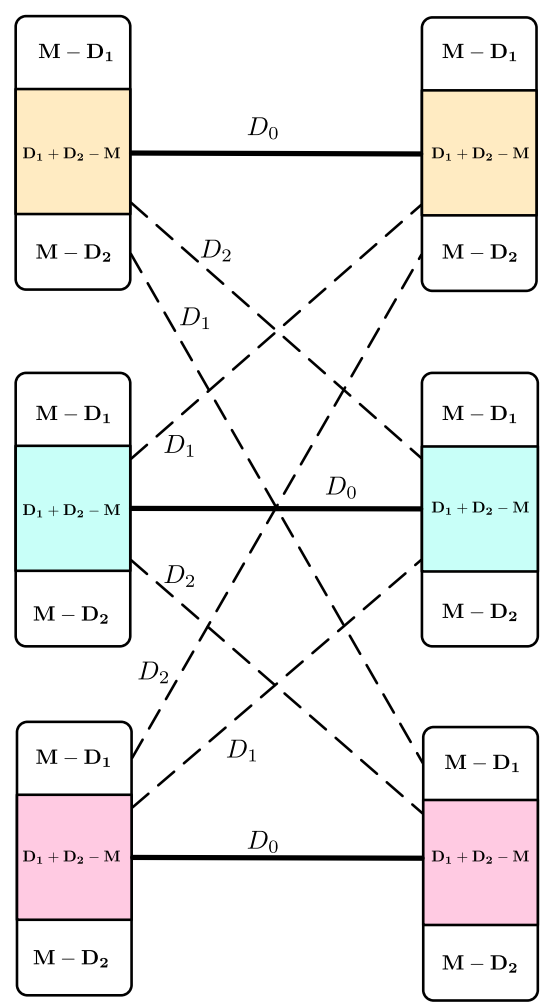

Fig. 6. Alignment in 3-user interference channel.

When direct channels are rank deficient, no more than $D_{0}$ vectors can be used for beamforming. For all values of $D_{0}$ such that $D_{0} \geq M-\frac{\min \left(M, D_{1}+D_{2}\right)}{2}$, same DoF can be obtained as in Table I by choosing specified number of beamforming vectors. When $D_{0}<M-\frac{\min \left(M, D_{1}+D_{2}\right)}{2}$, we send only $D_{0}$ beamforming vectors corresponding to all 3 cases, choosing first the zero-forcing vectors and then the alignment vectors as needed. In all cases, $\operatorname{dim}$ (Interference) + $\operatorname{dim}($ Desired $) \leq M$ since both desired and interference dimensions reduce with these changes.

Combining the DoF results for the 3 cases of Table I, achievability of $\min \left(D_{0}, M-\frac{\min \left(M, D_{1}+D_{2}\right)}{2}\right)$ DoF per user has been proved.

\section{B. Theorem 2: Proof of Outer Bound}

Converse proofs are described separately for the two cases: $D_{1}+D_{2}>M$ and $D_{1}+D_{2} \leq M$. For both cases, we first present the change of basis operations similar to [7], and then discuss the genie-aided outer bounds. We first present a lemma which is used for proving the outer bounds.

Lemma 1: For the $K$-user rank deficient interference channel, if a genie provides a subset of the noisy transmitted signals, denoted as $\mathcal{G}$, to Receiver $k$, then we can outer bound the mutual information term $I\left(W_{1}, W_{2}, \cdots, W_{K} ; Y_{k}^{n}, \mathcal{G}\right)$ as follows:

$$
\begin{aligned}
& I\left(W_{1}, \cdots, W_{K} ; Y_{k}^{n}, \mathcal{G}\right) \\
&= I\left(W_{1}, \cdots, W_{K} ; Y_{k}^{n}\right) \\
&+I\left(W_{1}, \cdots, W_{K} ; \mathcal{G} \mid Y_{k}^{n}\right)
\end{aligned}
$$

Channel $\bar{H}_{j i}^{\prime}-R_{j} H_{j i} T_{i}$

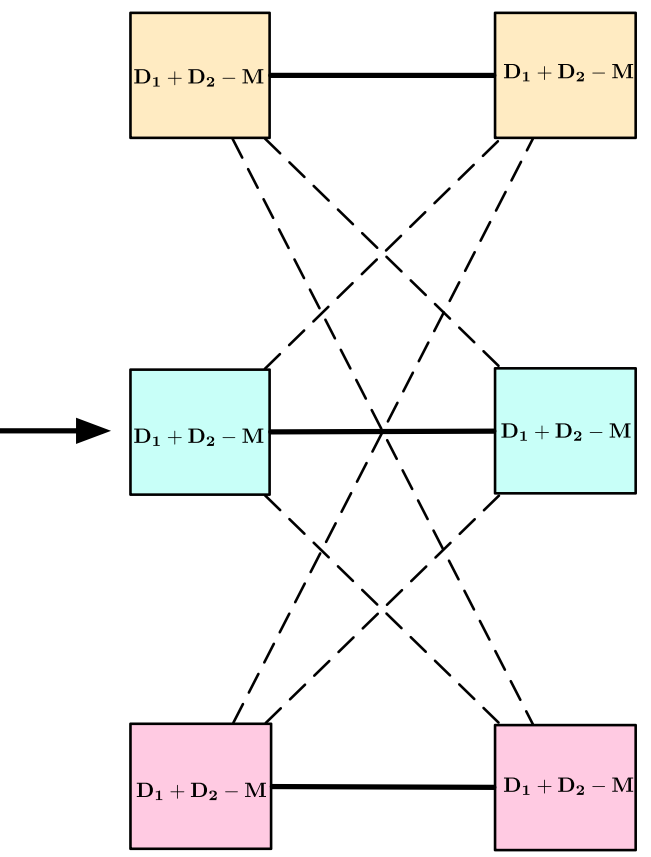

$$
\begin{aligned}
\leq & M n \log \rho+I\left(W_{1}, \cdots, W_{K} ; \mathcal{G} \mid Y_{k}^{n}\right) \\
& +n o(\log \rho) \\
\leq & M n \log \rho+h\left(\mathcal{G} \mid Y_{k}^{n}\right)-h\left(\mathcal{G} \mid Y_{k}^{n}, W_{1}, \cdots, W_{K}\right) \\
& +n o(\log \rho) \\
\leq & M n \log \rho+h\left(\mathcal{G} \mid Y_{k}^{n}\right)+n o(\log \rho) \\
= & M n \log \rho+h\left(\mathcal{G} \mid Y_{k}^{n}, W_{k}\right)+I\left(\mathcal{G} ; W_{k} \mid Y_{k}^{n}\right) \\
& +n o(\log \rho) \\
\leq & M n \log \rho+h\left(\mathcal{G} \mid Y_{k}^{n}, W_{k}\right) \\
& +n o(\log \rho)+o(n)
\end{aligned}
$$

Proof: In the derivations above, (15) follows from the mutual information chain rule. (16) is obtained because Receiver $k$ has only $M$ antennas. (17) follows from the definition of mutual information. (18) follows since given all $K$ messages, one can reconstruct the genie signals $\mathcal{G}$ within bounded noise distortion. (19) follows from the definition of mutual information, and (20) follows because of Fano's inequality, i.e., for a reliable coding scheme $H\left(W_{k} \mid Y_{k}^{n}\right) \leq o(n)$.

\section{B.1) Outer Bound When $D_{1}+D_{2}>M$ :}

\section{B.1.a) Change of basis:}

Step 1: For each receiver, a linear transformation $\mathbf{R}_{k}$ is designed such that the first $M-D_{2}$ antennas of Receiver $k$ do not hear Transmitter $k-1$ (left nullspace of $\mathbf{H}_{k(k-1)}$ ) and the last $M-D_{1}$ antennas of Receiver $k$ do not hear Transmitter $k+1$ (left nullspace of $\mathbf{H}_{k(k+1)}$ ). Corresponding signals seen at Receiver $k$ are denoted as $S_{k a}$ and $S_{k c}$, respectively. This is possible since $\operatorname{rank}\left(\mathbf{H}_{k(k+1)}\right)=D_{1}$ and $\operatorname{rank}\left(\mathbf{H}_{k(k-1)}\right)=D_{2}$. 


\begin{tabular}{r|r|}
\hline$\left|X_{1 a}\right|=M-D_{2}$ & $\circ$ \\
\hline$\left|X_{1 b}\right|=D_{1}+D_{2}-M>0$ & $\circ$ \\
\hline$\left|X_{1 c}\right|=M-D_{1}$ & $\circ$ \\
\hline
\end{tabular}

\begin{tabular}{r|c|}
\hline$\left|X_{2 a}\right|=M-D_{2}$ & $\circ$ \\
\hline$\left|X_{2 b}\right|=D_{1}+D_{2}-M>0$ & $\circ$ \\
\hline$\left|X_{2 c}\right|=M-D_{1}$ & $\circ$ \\
\hline
\end{tabular}

\begin{tabular}{r|c|}
\hline$\left|X_{3 a}\right|=M-D_{2}$ & $\circ$ \\
\hline$\left|X_{3 b}\right|=D_{1}+D_{2}-M>0$ & $\circ$ \\
\hline$\left|X_{3 c}\right|=M-D_{1}$ & $\circ$ \\
\hline
\end{tabular}

\begin{tabular}{|l|l|l}
\hline$\circ$ & $S_{1 a}\left(X_{2 a}\right)$ & $\left|S_{1 a}\right|=M-D_{2}$ \\
\hline$\circ$ & $S_{1 b}\left(X_{2 a}, X_{2 b}, X_{3 b}, X_{3 c}\right)$ & $\left|S_{1 b}\right|=D_{1}+D_{2}-M>0$ \\
\hline$\circ$ & $S_{1 c}\left(X_{3 c}\right)$ & $\left|S_{1 c}\right|=M-D_{1}$ \\
\hline
\end{tabular}

\begin{tabular}{|l|l|l}
\hline$\circ$ & $S_{2 a}\left(X_{3 a}\right)$ & $\left|S_{2 a}\right|=M-D_{2}$ \\
\hline$\circ$ & $S_{2 b}\left(X_{3 a}, X_{3 b}, X_{1 b}, X_{1 c}\right)$ & $\left|S_{2 b}\right|=D_{1}+D_{2}-M>0$ \\
\hline$\circ$ & $S_{2 c}\left(X_{1 c}\right)$ & $\left|S_{2 c}\right|=M-D_{1}$ \\
\hline
\end{tabular}

\begin{tabular}{|l|l|l}
\hline$\circ$ & $S_{3 a}\left(X_{1 a}\right)$ & $\left|S_{3 a}\right|=M-D_{2}$ \\
\hline$\circ$ & $S_{3 b}\left(X_{1 a}, X_{1 b}, X_{2 b}, X_{2 c}\right)$ & $\left|S_{3 b}\right|=D_{1}+D_{2}-M>0$ \\
\hline$\circ$ & $S_{3 c}\left(X_{2 c}\right)$ & $\left|S_{3 c}\right|=M-D_{1}$ \\
\hline
\end{tabular}

Fig. 7. Basis change for 3-user channel: $D_{1}+D_{2}>M$.

Step 2: In the $M$-dimensional space at Transmitter $k$, there is a $D_{1}$-dimensional subspace orthogonal to $M-D_{1}$ receiver antennas $(k-1) a$ and $D_{2}$-dimensional subspace orthogonal to $M-D_{2}$ receiver antennas $(k+1) c$. These two subspaces overlap in $I=D_{1}+D_{2}-M$ dimensions within the $M$-dimensional space seen by the transmitter, and these $I$ columns are chosen for matrix $\mathbf{T}_{k}$ at the transmitter, and the signal transmitted is denoted as $X_{k b}$. Other columns of $\mathbf{T}_{k}$ are chosen such that the first $M-D_{2}$ antennas of Transmitter $k$ are not heard by Receiver $k+1$ (right nullspace of $\mathbf{H}_{k(k-1)}$ ) and the last $M-D_{1}$ antennas of Transmitter $k$ are not heard by Receiver $k-1$ (right nullspace of $\mathbf{H}_{k(k+1)}$ ). Corresponding signals sent by Transmitter $k$ are denoted as $X_{k a}$ and $X_{k c}$, respectively.

Step 3: Remaining $D_{1}+D_{2}-M$ rows for linear transformation $\mathbf{R}_{k}$ are chosen so that they are linearly independent of other rows. Corresponding received signal is denoted as $S_{k b}$. Resulting network connectivity is shown in Figure 7.

B.1.b) Outer bound proof: Desired signal is assumed to be decodable and can be removed. Genie information to be given to Receiver 1 should include $2 M-\left(D_{1}+D_{2}\right)$ dimensions - $X_{2 c}^{n}, X_{3 a}^{n}$ which are not heard by receiver 1 . Receiver 1 has $M$ equations with $D_{1}+D_{2}$ unknowns. Hence only if genie information includes another $D_{1}+D_{2}-M$ dimensions, then at Receiver 1, there will be $M$ equations resolvable using $M$ unknowns.

Hence a genie provides (noisy versions of) $\mathcal{G}_{1}=\left\{X_{2 b}^{n}\right.$, $\left.X_{2 c}^{n}, X_{3 a}^{n}\right\}$ to Receiver 1 . The number of dimensions available to Receiver 1 is $M+\left|\mathcal{G}_{1}\right|=2 M$. With $2 M$ dimensions, Receiver 1 will be able to resolve both interfering signals and can decode all three messages, subject to the noise distortion. Since we are interested only in the DoF characterization, we will suppress the noise terms for compact notation (e.g., instead of $h\left(X^{n}+Z^{n}\right)$ we will simply write $h\left(X^{n}\right)$; see [7] for the details of the approach). Over $n$ channel uses, sum rate can be bounded as follows.

$$
\begin{aligned}
n R_{\Sigma} \leq & M n \log \rho+h\left(X_{2 b}^{n}, X_{2 c}^{n}, X_{3 a}^{n} \mid \bar{Y}_{1}^{n}\right) \\
& +n o(\log \rho)+o(n) \\
\leq & M n \log \rho+h\left(X_{3 a}^{n} \mid \bar{Y}_{1}^{n}\right)+h\left(X_{2 b}^{n} \mid \bar{Y}_{1}^{n}\right) \\
& +h\left(X_{2 c}^{n} \mid \bar{Y}_{1}^{n}, X_{2 b}^{n}, X_{3 a}^{n}\right)+n o(\log \rho)+o(n)
\end{aligned}
$$

$$
\begin{aligned}
\leq & M n \log \rho+h\left(X_{3 a}^{n}\right)+h\left(X_{2 b}^{n} \mid X_{2 a}^{n}\right) \\
& +h\left(X_{2 c}^{n} \mid X_{2 a}^{n}, X_{2 b}^{n}\right)+n o(\log \rho)+o(n) \\
= & M n \log \rho+h\left(X_{3 a}^{n}\right)+n R_{2}-h\left(X_{2 a}^{n}\right) \\
& +n o(\log \rho)+o(n)
\end{aligned}
$$

where (21) follows from Fano's inequality and Lemma 1. (22) follows from applying the chain rule. (23) follows since dropping condition terms cannot decrease differential entropy. Thus, we only keep $S_{1 a}^{n}$ as the condition term which is $X_{2 a}^{n}$. (24) is obtained because from the observations of $\left(X_{2 a}^{n}, X_{2 b}^{n}, X_{2 c}^{n}\right)$ we can decode $W_{2}$ subject to the noise distortion. By advancing user indices, and adding the resulting bounds, we have:

$$
3 n R_{\Sigma} \leq 3 M n \log \rho+n R_{\Sigma}+n o(\log \rho)+o(n)
$$

which implies that the DoF per user, $d \leq \frac{M}{2}$. Since $D_{0}$ is a known outer bound, we get $d \leq \min \left(D_{0}, \frac{M}{2}\right)$.

B.2) Outer Bound When $D_{1}+D_{2} \leq M$ :

\section{B.2.a) Change of basis:}

Step 1: For each receiver, a linear transformation $\mathbf{R}_{k}$ is designed such that the first $D_{1}$ antennas of Receiver $k$ do not hear Transmitter $k-1$ (left nullspace of $\mathbf{H}_{k(k-1)}$ ) and the last $D_{2}$ antennas of Receiver $k$ do not hear Transmitter $k+1$ (left nullspace of $\mathbf{H}_{k(k+1)}$ ). Corresponding signals seen at Receiver $k$ are denoted as $S_{k a}$ and $S_{k c}$, respectively. This is possible since $\operatorname{rank}\left(\mathbf{H}_{k(k+1)}\right)=D_{1}$ and rank $\left(\mathbf{H}_{k(k-1)}\right)=D_{2}$.

Step 2: In the $M$-dimensional space at Transmitter $k$, there is a $M-D_{1}$ dimensional subspace orthogonal to $D_{1}$ receiver antennas $(k-1) a$ and another $M-D_{2}$ dimensional subspace orthogonal to $D_{2}$ receiver antennas $(k+1) c$. These two subspaces have $I=M-\left(D_{1}+D_{2}\right)$ dimensional intersection at the transmitter, wherein $I$ columns are chosen for matrix $\mathbf{T}_{k}$, and the signal transmitted is denoted as $X_{k b}$. Then, we choose other columns of $\mathbf{T}_{k}$ such that $D_{1}$ antennas of Transmitter $k$ are not heard by Receiver $k+1$ (right nullspace of $\left.\mathbf{H}_{k(k-1)}\right)$ and $D_{2}$ antennas of Transmitter $k$ are not heard by Receiver $k-1$ (right nullspace of $\mathbf{H}_{k(k+1)}$ ). Corresponding signals sent by Transmitter $k$ are denoted as $X_{k a}$ and $X_{k c}$, respectively. 


\begin{tabular}{r|r|}
\hline$\left|X_{1 a}\right|=D_{1}$ & $\circ$ \\
\hline$\left|X_{1 b}\right|=M-\left(D_{1}+D_{2}\right) \geq 0$ & $\circ$ \\
\hline$\left|X_{1 c}\right|=D_{2}$ & $\circ$ \\
\hline
\end{tabular}

\begin{tabular}{r|c|}
\hline$\left|X_{2 a}\right|=D_{1}$ & $\circ$ \\
\hline$\left|X_{2 b}\right|=M-\left(D_{1}+D_{2}\right) \geq 0$ & $\circ$ \\
\hline$\left|X_{2 c}\right|=D_{2}$ & $\circ$ \\
\hline
\end{tabular}

\begin{tabular}{r|c|}
\hline$\left|X_{3 a}\right|=D_{1}$ & $\circ$ \\
\hline$\left|X_{3 b}\right|=M-\left(D_{1}+D_{2}\right) \geq 0$ & $\circ$ \\
\hline$\left|X_{3 c}\right|=D_{2}$ & $\circ$ \\
\hline
\end{tabular}

\begin{tabular}{|l|l|l}
\hline$\circ$ & $S_{1 a}\left(X_{2 a}\right)$ & $\left|S_{1 a}\right|=D_{1}$ \\
\hline$\circ$ & $S_{1 b}()$ & $\left|S_{1 b}\right|=M-\left(D_{1}+D_{2}\right) \geq 0$ \\
\hline$\circ$ & $S_{1 c}\left(X_{3 c}\right)$ & $\left|S_{1 c}\right|=D_{2}$ \\
\hline
\end{tabular}

\begin{tabular}{|l|l|l}
\hline$\circ$ & $S_{2 a}\left(X_{3 a}\right)$ & $\left|S_{2 a}\right|=D_{1}$ \\
\hline$\circ$ & $S_{2 b}()$ & $\left|S_{2 b}\right|=M-\left(D_{1}+D_{2}\right) \geq 0$ \\
\hline$\circ$ & $S_{2 c}\left(X_{1 c}\right)$ & $\left|S_{2 c}\right|=D_{2}$ \\
\hline
\end{tabular}

\begin{tabular}{|l|l|l}
\hline$\circ$ & $S_{3 a}\left(X_{1 a}\right)$ & $\left|S_{3 a}\right|=D_{1}$ \\
\hline$\circ$ & $S_{3 b}()$ & $\left|S_{3 b}\right|=M-\left(D_{1}+D_{2}\right) \geq 0$ \\
\hline$\circ$ & $S_{3 c}\left(X_{2 c}\right)$ & $\left|S_{3 c}\right|=D_{2}$ \\
\hline
\end{tabular}

Fig. 8. Basis change for 3-user channel: $D_{1}+D_{2} \leq M$.

Step 3: We consider only $D_{1}+D_{2}$ antennas at each receiver, remaining antennas are discarded since no signal is received (denoted as $S_{k b}$ ). Note that the desired signal is already assumed to be decoded and subtracted from the received signals. Resulting network connectivity is shown in Figure 8.

B.2.b) Outer bound proof: Desired signal is assumed to be decodable and can be removed. Genie information to be given to Receiver 1 should include $2 M-\left(D_{1}+D_{2}\right)$ dimensions - $X_{2 b}^{n}, X_{2 c}^{n}, X_{3 a}^{n}, X_{3 b}^{n}$ which are not heard by Receiver 1. Receiver 1 has $M$ equations with $D_{1}+D_{2}$ unknowns. Since $D_{1}+D_{2}<M$, choosing signal from only $D_{1}+D_{2}$ antennas results in $D_{1}+D_{2}$ equations becoming resolvable.

Hence a genie provides (noisy versions of $\mathcal{G}_{1}=\left\{X_{2 b}^{n}\right.$, $\left.X_{2 c}^{n}, X_{3 a}^{n}, X_{3 b}^{n}\right\}$ to Receiver 1, with which Receiver 1 will be able to resolve both interfering signals and can decode all three messages.

$$
\begin{aligned}
n R_{\sum} \leq & M n \log \rho+h\left(X_{2 b}^{n}, X_{2 c}^{n}, X_{3 a}^{n}, X_{3 b}^{n} \mid \bar{Y}_{1}^{n}\right) \\
& +n o(\log \rho)+o(n) \\
\leq & M n \log \rho+h\left(X_{3 a}^{n} \mid \bar{Y}_{1}^{n}\right)+h\left(X_{3 b}^{n} \mid \bar{Y}_{1}^{n}\right) \\
& +h\left(X_{2 b}^{n}, X_{2 c}^{n} \mid \bar{Y}_{1}^{n}, X_{3 a}^{n}, X_{3 b}^{n}\right)+n o(\log \rho)+o(n) \\
\leq & M n \log \rho+h\left(X_{3 a}^{n}\right)+h\left(X_{3 b}^{n}\right) \\
& +h\left(X_{2 b}^{n}, X_{2 c}^{n} \mid X_{2 a}^{n}\right)+n o(\log \rho)+o(n) \\
n R_{\sum}= & M n \log \rho+h\left(X_{3 a}^{n}\right)+h\left(X_{3 b}^{n}\right)+n R_{2}-h\left(X_{2 a}^{n}\right) \\
& +n o(\log \rho)+o(n) \\
\leq & M n \log \rho+h\left(X_{3 a}^{n}\right)+\left(M-\left(D_{1}+D_{2}\right)\right) n \log \rho \\
& +n R_{2}-h\left(X_{2 a}^{n}\right)+n o(\log \rho)+o(n)
\end{aligned}
$$

where (26) follows from Fano's inequality and Lemma 1. (27) follows from applying the chain rule. (28) follows since dropping condition terms cannot decrease differential entropy. Thus, we only keep $S_{1 a}^{n}$ as the condition term which is $X_{2 a}^{n}$. (29) is obtained because from the observations of $\left(X_{2 a}^{n}, X_{2 b}^{n}, X_{2 c}^{n}\right)$ we can decode $W_{2}$ subject to the noise distortion, (30) follows since the entropy of $X_{3 b}^{n}$ is constrained by $M-\left(D_{1}+D_{2}\right)$ antennas. By advancing user indices:

$$
3 n R_{\Sigma} \leq 3\left(2 M-\left(D_{1}+D_{2}\right)\right) n \log \rho+n R_{\Sigma}
$$

$$
+n o(\log \rho)+o(n)
$$

which implies that the DoF per user $d \leq \frac{2 M-\left(D_{1}+D_{2}\right)}{2}$. Since $D_{0}$ is a known outer bound, we get $d \leq \min \left(D_{0}, M-\frac{D_{1}+D_{2}}{2}\right)$. The converse result of Theorem 2 follows from the two cases described above.

\section{VI. $K$-USER CHANNEL}

For the $K$-user rank deficient interference channel, we assume all transmitters and receivers have $M$ antennas, all direct channels have rank $D_{0}$ and all cross channels have rank $D$. The DoF result is presented in the following theorem for time-varying channels.

Theorem 3: For the $K$-user rank deficient MIMO interference channel with $M$ antennas at each node, where the direct channels have rank $D_{0}$, cross channels have rank $D$ with time-varying channel coefficients, the DoF value per user is given by

$$
\frac{\operatorname{DoF}_{\Sigma}}{K}=\min \left\{D_{0}, M-\frac{\min (M,(K-1) D)}{2}\right\}
$$

Similar to the 3-user rank deficient interference channel, we note that for the $K$-user rank deficient interference channel, the rank-deficiency of direct channels does not help and the rank-deficiency of cross-channels does not hurt. Half-the-cake remains achievable as long as the direct channels support it.

In Figure 9, the optimal DoF value presented in Theorem 3 are compared with the achievable DoF established by Chae et al. in [23], assuming that each node has $M=10$ antennas and all direct and cross channels are of rank $D$. The green line corresponds to the optimal DoF while various dotted lines represent achievable DoF of [23] for different $K$. It can be noted that for this setting, optimal DoF per user being $\min \left(D, \frac{M}{2}\right)$ indicates that there is no DoF loss as number of users $(K)$ increases, which is not true for the result in [23].

While the nature of the DoF result remains consistent across rank-deficient 2-user, 3-user and $K$-user MIMO settings, the increasing complexity of achievable schemes requires increasingly sophisticated arguments to counter channel dependencies. The details of these arguments as well as the corresponding outer bounds are provided in the remainder of this work. 


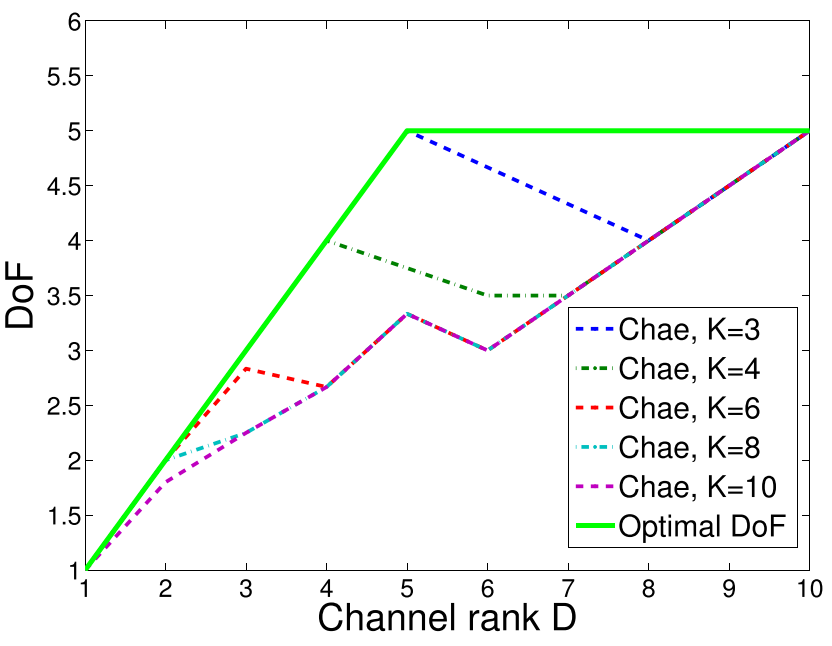

Fig. 9. DoF of $K$-user Rank Deficient Interference Channel with $M=10$ : Comparison with result of Chae at al. in [23].

\section{A. Alignment With Spatial Dependencies}

When all MIMO channels are full rank, the $K$-user MIMO channel can simply be decomposed to a $K M$ user SISO interference channel as shown in Figure 10. However, in the presence of rank deficiencies, there are spatial dependencies between some of the channels, due to which decomposition of the channel does not suffice and CJ scheme cannot be used directly. Hence, we perform one-sided decomposition of antennas at the transmitters, as in [21], while allowing joint processing at the receivers. We first discuss the CJ scheme tailored for channels with spatial dependencies and show that the DoF can be made arbitrarily close to half per user. Then we use the presented scheme for the $K$-user rank deficient channel with one-sided decomposition, to establish achievable DoF.

Let us consider the CJ scheme for $K$-user SISO channel $M_{k}=N_{k}=1, k \in\{1, \ldots, K\}$, with symbol extended channel over $n$ channel uses, such that all channel matrices $\mathbf{H}_{j i}$ are diagonal. We consider physical channels wherein the spatial dependencies do not change over time. Therefore, a spatial dependency which relates a set of channels, through an expression involving few generic channel variables, holds for all realizations of those generic channel variables. We denote the $N=K(K-1)$ linear transformations corresponding to the cross channels $\mathbf{H}_{j i}, i \neq j$ as $\mathbf{T}_{1}, \mathbf{T}_{2}, \ldots, \mathbf{T}_{N}$. Due to presence of rank deficient channels in the original network, there could be spatial dependencies between few of the cross channels. This could result in precoding matrix $\mathbf{V}_{n}$ not being full rank. In this section, we will show that spatial dependencies involving cross channels do not affect the achievable DoF adversely.

A.1) Interference Alignment: Let us denote the precoding matrix used at each transmitter in the original scheme as $\mathbf{V}_{n}$, and that used at each transmitter in presence of spatial dependencies by $\overline{\mathbf{V}}$ or $\overline{\mathbf{V}}_{n}$. Similar to construction in [5], we construct a precoding matrix $\overline{\mathbf{V}}$ such that it is invariant to the scaling factors $\mathbf{T}_{1}, \mathbf{T}_{2}, \ldots, \mathbf{T}_{N}$. Note that the commutative property of linear transformations $\mathbf{T}_{i}$ holds even in presence of spatial dependencies, which is necessary for aligning interference. This is because all $\mathbf{T}_{i}$ are diagonal channels resulting from symbol extensions.
We will now construct the precoding matrix $\overline{\mathbf{V}}_{n}$ by just removing dependent columns of $\mathbf{V}_{n}$. Similar to $\overline{\mathbf{V}}_{n}, \mathbf{V}_{n}$, we will use $\overline{\mathcal{I}}_{n}, \mathcal{I}_{n}$ to denote interference space at the receivers, with and without linearly dependent columns removed, respectively. Similar to [5], all transmitters use the same set of signaling vectors $\overline{\mathbf{V}}_{n}$ and all receivers approximately set aside the same subspace $\overline{\mathcal{I}}_{n}$ for interference.

$$
\begin{aligned}
\mathbf{V}_{n}= & \left\{\left(\mathbf{T}_{1}\right)^{\alpha_{1}}\left(\mathbf{T}_{2}\right)^{\alpha_{2}} \ldots\left(\mathbf{T}_{N}\right)^{\alpha_{N}} \mathbf{1}\right. \\
& \text { s.t. } \left.\sum_{i=1}^{N} \alpha_{i} \leq n, \alpha_{1}, \alpha_{2}, \ldots, \alpha_{N} \in \mathbb{Z}_{+} \cup\{0\}\right\} \\
\overline{\mathbf{V}}_{n}= & \text { Linearly independent columns of } \mathbf{V}_{n} \text { (Reordered) }
\end{aligned}
$$

wherein $\mathbf{1}$ refers to all-one column vector, and we choose $\mathcal{I}_{n}=\mathbf{V}_{n+1}$, and $\overline{\mathcal{I}}_{n}=\overline{\mathbf{V}}_{n+1}$.

In order to choose linearly independent columns, we first impose a lexicographic order on the columns of $\mathbf{V}_{n}$ and $\overline{\mathcal{I}}_{n}$, similar to that in [21]. All columns are arranged from left to right in increasing order of $\alpha_{1}$. Then columns corresponding to same order of $\alpha_{1}$ are arranged in increasing order of $\alpha_{2}$, and so on till $\alpha_{N}$. This ordering has the property that a tuple $\left(\alpha_{1}, \alpha_{2}, \ldots, \alpha_{N}\right)$ appears before the tuple $\left(\beta_{1}, \beta_{2}, \ldots, \beta_{N}\right)$, if and only if the first $\alpha_{i}$, which is different from $\beta_{i}$, is smaller than $\beta_{i}$. After reordering the columns in $\mathbf{V}_{n}$ and $\mathcal{I}_{n}$, each column is added sequentially starting from left to right, to $\overline{\mathbf{V}}_{n}$ and $\overline{\mathcal{I}}_{n}$, only if they are linearly independent with the columns that have been added already, in $\overline{\mathbf{V}}_{n}$ and $\overline{\mathcal{I}}_{n}$. Note that above reordering is only an exemplary choice for choosing linearly independent columns, and other choices exist.

Since we have removed only the dependent columns from $\mathbf{V}_{n}, \mathcal{I}_{n}$ to form $\overline{\mathbf{V}}_{n}, \overline{\mathcal{I}}_{n}$, the column spans of the precoding matrices remain the same.

$$
\begin{aligned}
& \operatorname{span}\left(\overline{\mathbf{V}}_{n}\right)=\operatorname{span}\left(\mathbf{V}_{n}\right) \\
& \operatorname{span}\left(\overline{\mathcal{I}}_{n}\right)=\operatorname{span}\left(\mathcal{I}_{n}\right)
\end{aligned}
$$

Construction of precoding matrices $\mathbf{V}_{n}, \mathcal{I}_{n}$ similar to that in [4] and [5] ensures that

$$
\begin{aligned}
& \operatorname{span}\left(\mathbf{T}_{i} \mathbf{V}_{n}\right) \subseteq \operatorname{span}\left(\mathcal{I}_{n}\right) \\
& \operatorname{span}\left(\mathbf{T}_{i} \overline{\mathbf{V}}_{n}\right)=\operatorname{span}\left(\mathbf{T}_{i} \mathbf{V}_{n}\right)
\end{aligned}
$$

Thus, we have aligned interference from all unintended transmitters at the receivers in space $\overline{\mathcal{I}}_{n}$,

$$
\operatorname{span}\left(\mathbf{T}_{i} \overline{\mathbf{V}}_{n}\right)=\operatorname{span}\left(\mathbf{T}_{i} \mathbf{V}_{n}\right) \subseteq \operatorname{span}\left(\mathcal{I}_{n}\right)=\operatorname{span}\left(\overline{\mathcal{I}}_{n}\right)
$$

In the original construction, number of precoding vectors was given by $\left|\mathbf{V}_{n}\right|=\left(\begin{array}{c}n+N \\ N\end{array}\right)$ and $\left|\mathcal{I}_{n}\right|=\left(\begin{array}{c}n+N+1 \\ N\end{array}\right)$. While we do not specify the number of precoding vectors in $\overline{\mathbf{V}}_{n}, \overline{\mathcal{I}}_{n}$, we know that $\left|\overline{\mathbf{V}}_{n}\right|<\left|\overline{\mathcal{I}}_{n}\right|=\left|\overline{\mathbf{V}}_{n+1}\right|$.

We have so far shown that all interference signals align in the span of $\overline{\mathcal{I}}_{n}$ at the receivers, in the presence of spatial dependencies. Note that this is possible because we assume all spatial dependencies to involve only the cross channels. Now we will show that desired and interference signal spaces 

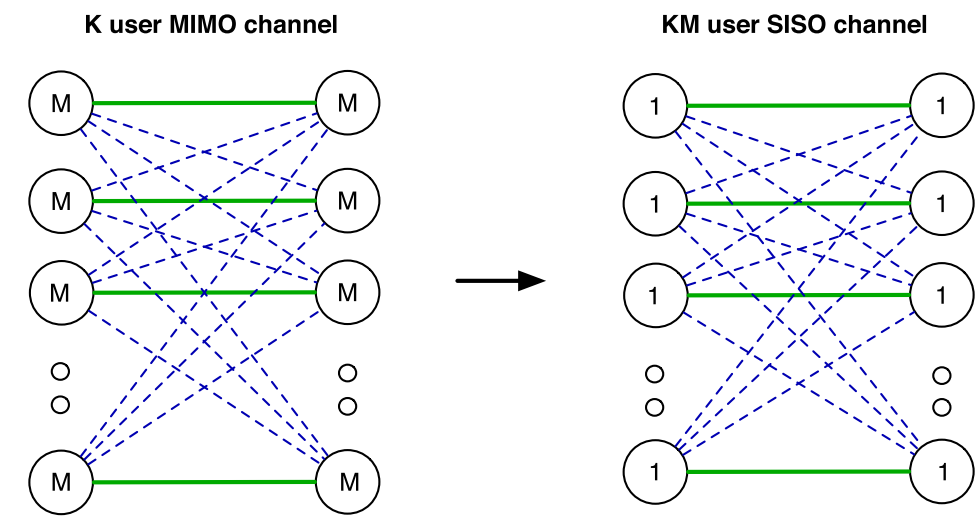

Fig. 10. $K$-user interference channel: Decomposition.

can occupy half the dimensions each at all receivers, almost surely.

A.2) Half the Cake: In following proofs, we use limit infimum as defined below, since for sequences whose convergence is not guaranteed, limits may not exist.

Definition (Limit Infimum): The limit infimum (lim inf) of a sequence $x_{n}$ is the largest real number $b$ that, for any positive real number $\epsilon$, there exists a natural number $N$ such that $x_{n}>$ $b-\epsilon$ for all $n>N$.

Property: For sequence $x_{n}$, if $a>\lim \inf x_{n}$, then there is an infinite subsequence $x_{n_{k}}$ of $x_{n}$ such that $a>x_{n_{k}} \forall k$.

Lemma 2: For the $K$-user interference channel with spatial dependencies, and precoding matrices with linearly independent columns, denoted as $\overline{\mathbf{V}}_{n}$ and $\overline{\mathcal{I}}_{n}=\overline{\mathbf{V}}_{n+1}$,

i. $\liminf _{n \rightarrow \infty} \frac{\left|\overline{\mathbf{V}}_{n+1}\right|-\left|\overline{\mathbf{V}}_{n}\right|}{\left|\overline{\mathbf{V}}_{n}\right|}=0$

ii. There exist a subsequence of $n$ such that $\frac{\left|\overline{\mathbf{V}}_{n}\right|}{\left|\overline{\mathbf{V}}_{n}\right|+\left|\overline{\mathcal{I}}_{n}\right|}$ can be made arbitrarily close to $\frac{1}{2}$

Proof: i. We will prove this by contradiction. Suppose the contrary is true, i.e., there exists a positive number $\epsilon>0$ such that

$$
\liminf _{n \rightarrow \infty} \frac{\left|\overline{\mathbf{V}}_{n+1}\right|-\left|\overline{\mathbf{V}}_{n}\right|}{\left|\overline{\mathbf{V}}_{n}\right|}>\epsilon
$$

which can be written as

$$
\liminf _{n \rightarrow \infty} \frac{\left|\overline{\mathbf{V}}_{n+1}\right|}{\left|\overline{\mathbf{V}}_{n}\right|}>(1+\epsilon)
$$

Considering the definition of limit infimum, above relation implies that there exists a positive integer $n_{0}$ such that for all $n>n_{0}$, following holds.

$$
\frac{\left|\overline{\mathbf{V}}_{n+1}\right|}{\left|\overline{\mathbf{V}}_{n_{0}}\right|}>(1+\epsilon)^{n+1-n_{0}}
$$

Above is a recursive relation that holds for all positive integers $n$. Therefore we deduce that

$$
\left|\overline{\mathbf{V}}_{n}\right|>(1+\epsilon)^{n-n_{0}}\left|\overline{\mathbf{V}}_{n_{0}}\right|
$$

Based on construction of precoding vectors in the CJ scheme, we know that

$$
\left|\overline{\mathbf{V}}_{n+1}\right| \leq\left(\begin{array}{c}
n+N+1 \\
N
\end{array}\right)
$$

Hence, we have the following

$$
\frac{\left|\overline{\mathbf{V}}_{n+1}\right|}{\left|\overline{\mathbf{V}}_{n}\right|} \leq \frac{\left(\begin{array}{c}
n+N+1 \\
N
\end{array}\right)}{(1+\epsilon)^{n-n_{0} \overline{\mathbf{V}}_{n_{0}}}}
$$

It can be seen that for large $n$, term on right goes to zero since it is a ratio of a polynomial over an exponential in $n$. However, this cannot be true since $\left|\overline{\mathbf{V}}_{n}\right| \leq\left|\overline{\mathbf{V}}_{n+1}\right|$, leading to a contradiction.

Hence the assumption in (42) cannot hold, and we have proved (40), i.e., growth rate of size of precoding matrix after removing the dependent columns, reaches zero asymptotically for large $n$. In other words, $\overline{\mathbf{V}}_{n+1}$ and $\overline{\mathbf{V}}_{n}$ are "almost" of the same size. ii. From i., note that

$$
\liminf _{n \rightarrow \infty} \frac{\left|\overline{\mathbf{V}}_{n+1}\right|}{\left|\overline{\mathbf{V}}_{n}\right|}=1
$$

Also, for sequence $x_{n}$, if $a>\liminf x_{n}$, then there is an infinite subsequence $x_{n_{k}}$ of $x_{n}$ such that $a>x_{n_{k}} \forall k$. Using this, we can choose $n, \delta$ such that following holds

$$
\frac{\left|\overline{\mathbf{V}}_{n+1}\right|}{\left|\overline{\mathbf{V}}_{n}\right|}<1+\delta
$$

From above relation, we can deduce the best value of

$$
\frac{\left|\overline{\mathbf{V}}_{n}\right|}{\left|\overline{\mathbf{V}}_{n}\right|+\left|\overline{\mathcal{I}}_{n}\right|}=\frac{\left|\overline{\mathbf{V}}_{n}\right|}{\left|\overline{\mathbf{V}}_{n+1}\right|+\left|\overline{\mathbf{V}}_{n}\right|} \approx \frac{1}{1+(1+\delta)}=\frac{1}{2+\delta}
$$

Hence with appropriate choice for $\delta$, ratio of desired signal dimensions and total signal dimensions can be made arbitrarily close to $\frac{1}{2}$ for large $n$.

We will now use above lemma to establish the achievable DoF value for the $K$-user rank deficient interference channel.

\section{B. Theorem 3: Proof of Achievability}

Achievability proofs for $K$-user rank deficient channel with time-varying channel coefficients, are presented separately for two regions -

- Sum of cross channel ranks, $(K-1) D \leq$ Number of antennas, $M$

- Sum of cross channel ranks, $(K-1) D>$ Number of antennas, $M$

Achievable scheme involves only zero-forcing when $(K-1) D \leq M$ (Region 1). The CJ scheme with one-sided decomposition is involved when $(K-1) D>M$ (Region 2). 


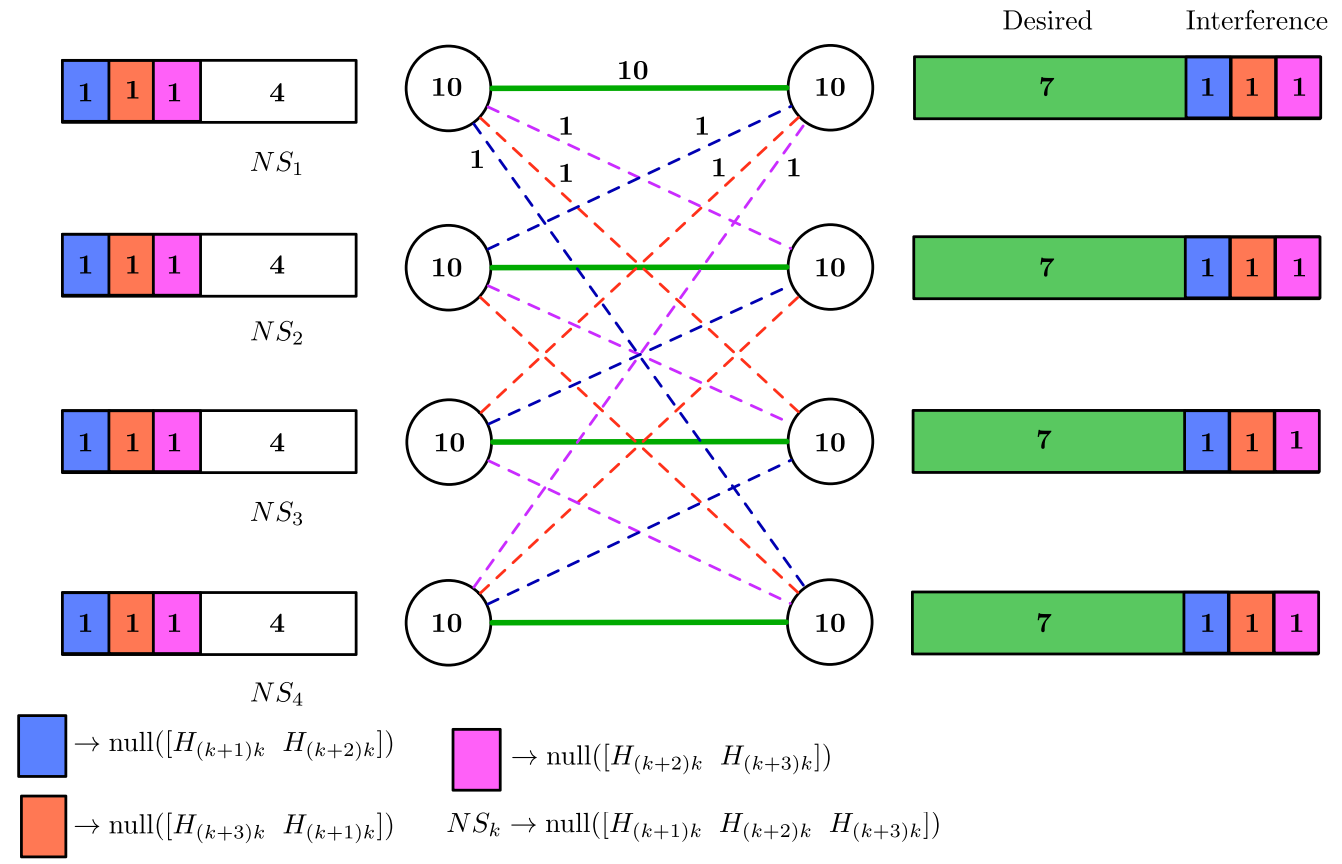

Fig. 11. Achievability: Example setting for $(K-1) D \leq M$.

B.1) Region 1 (Interference Spans Part of the Receiver Signal Space, $(\mathbf{K}-\mathbf{1}) \mathbf{D} \leq \mathbf{M})$ : We will first consider all direct channels to be full rank, and show that zero-forcing is sufficient to achieve DoF of $M-\frac{(K-1) D}{2}$ per user.

Since all cross channels are of rank $D$, the common nullspace of all cross channels at each transmitter has $M-(K-1) D$ dimensions. Hence, each transmitter can choose $M-(K-1) D$ zero-forcing beamforming vectors from the common nullspace such that these vectors do not cause interference at any of the $K-1$ unintended receivers. For example, Transmitter 1 chooses $M-(K-1) D$ vectors from the following nullspace, so that no interference is caused at the receivers $2,3, \ldots, K$.

$$
\operatorname{null}\left(\left[\begin{array}{llll}
\mathbf{H}_{21} & \mathbf{H}_{31} & \cdots & \mathbf{H}_{K 1}
\end{array}\right]\right)
$$

Additionally, $\frac{(K-1) D}{2}$ vectors can be chosen from the common nullspaces of $K-2$ cross channels. This is possible because apart from $M-(K-1) D$ dimensions already chosen, there are $(K-1) D$ dimensions in the set of common nullspaces of $K-2$ cross channels at each transmitter. For example, Transmitter 1 chooses $\frac{(K-1) D}{2}$ vectors, with $\frac{D}{2}$ vectors from each of the following nullspaces.

$$
\begin{aligned}
& \operatorname{null}\left(\left[\begin{array}{llll}
\mathbf{H}_{31} & \mathbf{H}_{41} & \cdots & \mathbf{H}_{K 1}
\end{array}\right]\right) \\
& \operatorname{null}\left(\left[\begin{array}{llll}
\mathbf{H}_{21} & \mathbf{H}_{41} & \cdots & \mathbf{H}_{K 1}
\end{array}\right]\right) \\
& \operatorname{null}\left(\left[\begin{array}{llll}
\mathbf{H}_{21} & \mathbf{H}_{31} & \cdots & \mathbf{H}_{(K-1) 1}
\end{array}\right]\right)
\end{aligned}
$$

Hence at each transmitter, we choose $M-(K-1) D$ beamforming vectors such that any receiver does not see interference and another $\frac{(K-1) D}{2}$ vectors are chosen such that each receiver sees only $\frac{(K-1) D}{2}$ dimensions of interference from all unintended transmitters. Note that all cross channels in this section $\left(\mathbf{H}_{j i}\right)$ are $M \times M$ matrices of rank $D$, corresponding to current channel realization $n$.

Since each unintended receiver sees only $D$ signal dimensions from a transmitter which do not overlap, $\frac{D}{2}$ vectors are chosen from signal space seen by each of the $K-1$ unintended receivers. As a result, each receiver sees interference of only $\frac{(K-1) D}{2}$ dimensions, and so desired symbols are resolvable since the number of signal dimensions are given as

$$
\begin{aligned}
\operatorname{dim}(\text { Desired }) & =M-\frac{(K-1) D}{2} \\
\operatorname{dim}(\text { Interference }) & =\frac{(K-1) D}{2}
\end{aligned}
$$

As an illustrative example, let us consider 4-user rank deficient interference channel to describe the beamforming vector choices, as shown in Figure 11. In this example, each node has $M=10$ antennas with all direct channels of rank $D_{0}=M$, and all cross channels of rank $D=2$, so that $(K-1) D=6<M .4$ beamforming vectors can be chosen from the common nullspace of all 3 cross channels at each transmitter, denoted as $N S_{k}$. Another 3 dimensions are chosen at Transmitter 1 as follows: The common nullspace of channels $\mathbf{H}_{21}, \mathbf{H}_{31}$ has 2 dimensions, and we choose one generic vector from this space. The common nullspace of channels $\mathbf{H}_{31}, \mathbf{H}_{41}$ has 2 dimensions, and we choose one vector from this space. The common nullspace of channels $\mathbf{H}_{21}, \mathbf{H}_{41}$ has 2 dimensions, and we choose one vector from this space. Similarly 3 vectors can be chosen at transmitters 2, 3, 4 from corresponding common nullspaces. Hence at each transmitter, we choose 4 beamforming vectors such that they will not cause interference at any receiver, and 3 beamforming vectors are chosen so that each receiver sees only 3-dimensional interference. Hence desired signal occupying 7 dimensions is resolvable from 3-dimensional interference at all receivers. 
When $D$ is odd, 2 symbol extensions of the channel are used to achieve DoF of $M-\frac{(K-1) D}{2}$.

Note that the above result holds for other direct channel ranks when $D_{0} \geq M-\frac{(K-1) D}{2}$. When direct channels are of rank $D_{0}<M-\frac{(K-1) D}{2}$, the direct channel becomes the bottleneck and only $D_{0}$ DoF per user are achievable.

B.2) Region 2 (Interference Spans the Full Receiver Signal Space, $(\mathbf{K}-\mathbf{1}) \mathbf{D}>\mathbf{M})$ :

B.2.a) Ergodic interference alignment: For the region $(K-1) D>M$, we first discuss the achievable scheme through ergodic interference alignment with time-varying channel coefficients, similar to the scheme in [27]. All symbols are repeated by the $K$ transmitters over 2 channel uses $t_{1}$ and $t_{2}$, where all cross-channels remain the same $\mathbf{H}_{j i}\left(t_{1}\right)=$ $\mathbf{H}_{j i}\left(t_{2}\right), i \neq j$, but all direct channels are different $\mathbf{H}_{i i}\left(t_{1}\right) \neq$ $\mathbf{H}_{i i}\left(t_{2}\right)$. All receivers subtract the symbols received at channel use $t_{1}$ from the symbols received at channel use $t_{2}$. Interference is eliminated since it was the same during both channel uses $t_{1}$ and $t_{2}$. Desired signals remain because direct channels changed into new generic channels between the 2 channel uses. Note that the ranks of the cross channels do not impact this achievable scheme. Thus, $M$ independent equations in $M$ desired variables are obtained over 2 channel uses, achieving $\frac{M}{2}$ DoF per user, when direct channel rank $D_{0} \geq \frac{M}{2}$. This scheme is similar to coding over a channel matrix and its complement, like in ergodic interference alignment of [28], but is more general since there are no assumptions on the channel phase. It is straightforward to extend the scheme to other direct channel ranks, i.e., when $D_{0}<\frac{M}{2}$, and show that achievable DoF per user is $\min \left(D_{0}, \frac{M}{2}\right)$.

While the ergodic interference alignment scheme helps in establishing the DoF of the rank deficient channel, it does so only for channel coefficients exhibiting the ergodic nature, which stem from the requirement for all cross channel coefficients to repeat. Hence, we avoid making such restrictive assumptions and consider channels without the ergodic nature, since in practice, channel fading distribution could change over time. With this premise, henceforth, we prove the same DoF result using asymptotic interference alignment (CJ) scheme. Further, asymptotic schemes often serve as stepping stones to translate DoF results obtained for time-varying channels to constant channels, using real alignment schemes, as described in [17] and [29].

B.2.b) Asymptotic interference alignment: We now discuss CJ scheme over symbol extended channel with timevarying channel coefficients, by performing decomposition of antennas only at the transmitters (i.e., no joint processing) for the region $(K-1) D>M$. The idea of one-sided decomposability was earlier used by Sun et al. in [21] for X channel to prove linear independence of desired and interfering signals at the receivers. From Section VI-A, we infer that the precoding matrix $\overline{\mathbf{V}}_{n}$ could be made full rank, by discarding the linearly dependent columns of $\mathbf{V}_{n}$. Also, Lemma 2 implies that the ratio of desired signal dimensions over total signaling dimensions can be made arbitrarily close to $\frac{1}{2}$, after discarding the linearly dependent columns.
To establish achievable DoF, we also need to show that the desired and interfering signals are linearly independent at all receivers, i.e., we need to show $\left[\begin{array}{lll}\mathbf{H}_{k k} \overline{\mathbf{V}}_{n} & \mathbf{H}_{k j} \overline{\mathbf{V}}_{n}\end{array}\right]$ is full rank for all $k, j \in\{1, \ldots, K M\}, j \neq k$ wherein $\mathbf{H}_{k j}$ represents the channel between Transmitter $j$ and Receiver $k$. Decomposition of antennas at all nodes would not help if direct channels are rank deficient, since there are dependencies between the direct and the cross channels. Hence, we perform one-sided decomposition of the channel, wherein we treat antennas of each transmitter node separately while allowing joint processing at the receivers. We first consider all direct channels to be of rank $D_{0} \geq \frac{M}{2}$, and show that DoF of $\frac{M}{2}$ per user can be achieved. When $D_{0}<\frac{M}{2}$, it can be shown that DoF of $D_{0}$ per user can be achieved, establishing that the achievable DoF per user is $\min \left(D_{0}, \frac{M}{2}\right)$ for the region $(K-1) D>M$. We describe the proofs for even $M$, and symbol extensions are used if necessary, for odd $M$ or other cases.

Let us consider the $K$-user rank deficient interference channel wherein all direct channels are of rank $D_{0} \geq \frac{M}{2}$. With one-sided decomposition of the channel, there are $M K$ transmitters each with single antenna, sending messages to $K$ receivers each with $M$ antennas. Consider $n$ symbol extension of the original channel so that each transmitter sees an $n$-dimensional signal space while each receiver has $n M$ dimensional signal space. The value of $n$ will be specified later. The input-output relationship of the symbol-extended channel is

$$
\begin{aligned}
Y^{[j]}(\kappa) & =\sum_{i=1}^{M K} \mathbf{H}^{[j i]}(\kappa) X^{[i]}(\kappa)+Z^{[j]}(\kappa) \\
& =\sum_{i=1}^{M K}\left[\begin{array}{c}
\tilde{\mathbf{H}}_{1}^{[j i]}(\kappa) \\
\vdots \\
\tilde{\mathbf{H}}_{M}^{[j i]}(\kappa)
\end{array}\right] X_{j \in\{1,2, \ldots, K\}}^{[i]}(\kappa)+Z^{[j]}(\kappa),
\end{aligned}
$$

where $X^{[i]}(\kappa) \in \mathbb{C}^{n \times 1}$ is the signal vector sent by the $i^{\text {th }}$ transmitter and $Y^{[j]}(\kappa) \in \mathbb{C}^{n M \times 1}$ is the received signal vector at Receiver $j$ over extended channel-use index $\kappa$. $\tilde{\mathbf{H}}_{m}^{[j i]}(\kappa) \in \mathbb{C}^{n \times n}$ represents the diagonal channel matrix from Transmitter $i$ to the $m^{\text {th }}$ receive antenna of Receiver $j$ where $m \in\{1, \ldots, M\}$, i.e.,

$$
\tilde{\mathbf{H}}_{m}^{[j i]}(\kappa)=\left[\begin{array}{ccc}
H_{m}^{[j i]}(n(\kappa-1)+1) & \cdots & 0 \\
\vdots & \ddots & \vdots \\
0 & \cdots & H_{m}^{[j i]}(n \kappa)
\end{array}\right]
$$

The channel-use index is suppressed for compactness. Each transmitter selects the same beamforming matrix $\mathbf{V}$ to precode its message for Receiver $j \in\{1, \ldots, K\}$. Specifically, $\mathbf{X}^{[i]}=\mathbf{V} \mathbf{x}^{[i]}, i \in\{1, \ldots, M K\}$, where $\mathbf{V}$ is the $n \times\left(\begin{array}{c}n+N \\ N\end{array}\right)$ precoding matrix and $\mathbf{x}^{[i]}$ is the $|\mathbf{V}| \times 1$ data stream vector from Transmitter $i$. In order to consolidate the interference caused by $\mathbf{V}$ at all receivers $j \in\{1, \ldots, K\}$ as much as possible, we set the interference space brought by $\mathbf{V}$ at receivers $1, \ldots, K$ 
to roughly $\underbrace{\mathbf{V} \times \cdots \times \mathbf{V}}_{M \text { times }}$, in which interference will be aligned

$$
\begin{aligned}
& \operatorname{span}\left[\tilde{\mathbf{H}}^{[j i]} \mathbf{V}\right]= \operatorname{span}\left[\begin{array}{c}
\tilde{\mathbf{H}}_{1}^{[j i]} \mathbf{V} \\
\tilde{\mathbf{H}}_{2}^{[j i]} \mathbf{V} \\
\vdots \\
\tilde{\mathbf{H}}_{M}^{[j i]} \mathbf{V}
\end{array}\right] \\
& \cong \operatorname{span}\left[\begin{array}{cccc}
\mathbf{V} & \mathbf{0} & \cdots & \mathbf{0} \\
\mathbf{0} & \mathbf{V} & \cdots & \mathbf{0} \\
\vdots & \vdots & \ddots & \vdots \\
\mathbf{0} & \mathbf{0} & \cdots & \mathbf{V}
\end{array}\right]_{n M \times|\mathbf{V}| M} \\
& i \in\{1, \ldots, M K\}, \\
& j \in\{1, \ldots, K\}, \quad j \neq\left\lfloor\frac{i-1}{M}\right\rfloor+1
\end{aligned}
$$

All of the above conditions can be written as

$$
\begin{aligned}
\mathbf{V} \approx & \tilde{\mathbf{H}}_{m}^{[j i]} \mathbf{V}, \\
& i \in\{1, \ldots, M K\}, \quad j \in\{1, \ldots, K\}, \\
& j \neq\left\lfloor\frac{i-1}{M}\right\rfloor+1, \quad m \in\{1, \ldots, M\}
\end{aligned}
$$

where $\cong, \approx$ are used to denote that $\mathbf{V}$ is approximately invariant to the scaling factors $\tilde{\mathbf{H}}_{m}^{[j i]}$. To paraphrase, messages for Receiver $j$ are sent along the same signal space $\mathbf{V}$ and aligned into $\mathbf{V} \times \cdots \times \mathbf{V}$ space at all receivers $l \in\{1, \ldots, j-1$, $j+1, \ldots, K\}$.

Let us define $\mathcal{I}=\operatorname{span}\left(\bigcup_{i, l, m} \operatorname{span}\left(\tilde{\mathbf{H}}_{m}^{[l i]} \mathbf{V}\right)\right)$, which is the span of union of interference terms caused by $\mathbf{V}$ on antenna $m$ at all receivers other than the intended receiver, and condition (56) becomes $\mathbf{V} \approx \mathcal{I}$ which essentially states that $\mathbf{V}$ scales invariantly by the interference-carrying links. It can be satisfied simultaneously with the CJ scheme using beamforming vectors:

$$
\begin{aligned}
\mathbf{V}=\{ & \left\{\prod_{i, j, m}\left(\tilde{\mathbf{H}}_{m}^{[j i]}\right)^{\alpha_{m}^{[j i]}}\right) \mathbf{1}, \quad \text { s. t. } \sum_{i, j, m} \alpha_{m}^{[j i]} \leq n, \\
& \alpha_{m}^{[j i]} \in\{0\} \cup \mathbb{Z}_{+}, \quad i \in\{1, \ldots, M K\}, \\
& \left.j \in\{1, \ldots, K\}, \quad j \neq\left\lfloor\frac{i-1}{M}\right\rfloor+1, m \in\{1, \ldots, M\}\right\},
\end{aligned}
$$

$$
\begin{aligned}
\mathcal{I}= & \left\{\left(\prod_{i, j, m}\left(\tilde{\mathbf{H}}_{m}^{[j i]}\right)^{\alpha_{m}^{[j i]}}\right) \mathbf{1}, \quad \text { s. t. } \sum_{i, j, m} \alpha_{m}^{[j i]} \leq n+1,\right. \\
& \alpha_{m}^{[j i]} \in\{0\} \cup \mathbb{Z}_{+}, \quad i \in\{1, \ldots, M K\}, \\
& \left.j \in\{1, \ldots, K\}, \quad j \neq\left\lfloor\frac{i-1}{M}\right\rfloor+1, m \in\{1, \ldots, M\}\right\}
\end{aligned}
$$

where 1 is the $n \times 1$ all ones column vector.

Thus $\mathbf{V}$ contains product terms up to degree $n$ and interference term $\mathcal{I}$ contains product terms up to degree $n+1$. Note that the original network had rank deficient channels which introduces spatial dependencies, however, we discard all linearly dependent columns of $\mathbf{V}$ and $\mathcal{I}$ after reordering the columns in a lexicographic order, as discussed in Section VI-A. We represent the resultant matrices after discarding all linearly dependent columns, as $\overline{\mathbf{V}}$ and $\overline{\mathcal{I}}$. Unlike in Section VI-A, some of the cross channels are not included in the construction of precoding matrix above, which is beneficial for the linear independence proofs. However, this does not violate the result of Lemma 2.

At each receiver, desired signals occupy $M|\overline{\mathbf{V}}|$ dimensions and aligned interference occupies $M|\overline{\mathcal{I}}|$ dimensions. To accommodate both desired signals and interference, the size of receive signal space, $n M$, should be as big as the sum of the dimensions of desired signals and interference. Therefore, we set $n M=M|\overline{\mathbf{V}}|+M|\overline{\mathcal{I}}|$, i.e., $n=|\overline{\mathbf{V}}|+|\overline{\mathcal{I}}|$. To ensure decodability, we should guarantee the linear independence of the desired signals from interference.

Due to symmetry, we only prove linear independence of signals at Receiver 1 . Let us define

$$
\mathbf{D}_{m}^{[1]}=\left[\tilde{\mathbf{H}}_{m}^{[11]} \overline{\mathbf{V}} \ldots \tilde{\mathbf{H}}_{m}^{[1 M]} \overline{\mathbf{V}}\right], \quad m \in\{1, \ldots, M\}
$$

which corresponds to the desired signal at the $m^{\text {th }}$ antenna of Receiver 1 . Then the desired signal at Receiver 1 correspond to the columns of $\mathbf{D}^{[1]}$.

$$
\mathbf{D}^{[1]}=\left[\begin{array}{c}
\mathbf{D}_{1}^{[1]} \\
\mathbf{D}_{2}^{[1]} \\
\vdots \\
\mathbf{D}_{M}^{[1]}
\end{array}\right]=\left[\begin{array}{cccc}
\tilde{\mathbf{H}}_{1}^{[11]} \overline{\mathbf{V}} & \cdots & \tilde{\mathbf{H}}_{1}^{[1 M]} \overline{\mathbf{V}} \\
\tilde{\mathbf{H}}_{2}^{[11]} \overline{\mathbf{V}} & \cdots & \tilde{\mathbf{H}}_{2}^{[1 M]} \overline{\mathbf{V}} \\
\vdots & \ddots & \vdots \\
\tilde{\mathbf{H}}_{M}^{[11]} & \cdots & \tilde{\mathbf{H}}_{M}^{[1 M]} \overline{\mathbf{V}}
\end{array}\right]
$$

At Receiver 1, interference from transmitters $j \in$ $\{2, \ldots, K\}$, are aligned in the column span of

$$
\mathbf{E}^{[1]}=\left[\begin{array}{cccc}
\overline{\mathcal{I}} & \mathbf{0} & \ldots & \mathbf{0} \\
\mathbf{0} & \overline{\mathcal{I}} & \ldots & \mathbf{0} \\
\vdots & \vdots & \ddots & \vdots \\
\mathbf{0} & \mathbf{0} & \cdots & \overline{\overline{\mathcal{I}}}
\end{array}\right]=\mathbf{I}_{M} \otimes \overline{\mathcal{I}}
$$

We need to show that the $n M \times n M$ matrix $\mathbf{F}^{[1]}=\left[\mathbf{D}^{[1]} \mathbf{E}^{[1]}\right]$ has full rank almost surely. We will first show that the desired signals are linearly independent among themselves and then prove that the desired signal space does not overlap with the interference space.

Step 1: We first prove that the desired signals are linearly independent, i.e., the $n M \times|\overline{\mathbf{V}}| M$ matrix $\mathbf{D}^{[1]}$ has full rank, almost surely. To do this, it is sufficient to prove the following $M|\overline{\mathbf{V}}| \times M|\overline{\mathbf{V}}|$ submatrix of $\mathbf{D}^{[1]}$ is full rank.

$$
\overline{\mathbf{D}}^{[1]}=\left[\begin{array}{c}
\overline{\mathbf{D}}_{a}^{[1]} \\
\overline{\mathbf{D}}_{b}^{[1]}
\end{array}\right]_{M|\overline{\mathbf{V}}| \times M|\overline{\mathbf{V}}|}
$$

where

$\overline{\mathbf{D}}_{a}^{[1]}$ has the top $|\overline{\mathbf{V}}|$ rows of each $\mathbf{D}_{m}^{[1]}, \quad m \in\left\{1, \ldots, \frac{M}{2}\right\}$

$\overline{\mathbf{D}}_{b}^{[1]}$ has the bottom $|\overline{\mathbf{V}}|$ rows of each $\mathbf{D}_{m}^{[1]}$,

$$
m \in\left\{\frac{M}{2}+1, \ldots, M\right\}
$$


and so, $\overline{\mathbf{D}}^{[1]}$ can be written as

$$
\overline{\mathbf{D}}^{[1]}=\left[\begin{array}{cccc}
\tilde{\mathbf{H}}_{1 a}^{[11]} \overline{\mathbf{V}}_{\mathbf{a}} & \tilde{\mathbf{H}}_{1 a}^{[12]} \overline{\mathbf{V}}_{\mathbf{a}} & \cdots & \tilde{\mathbf{H}}_{1 a}^{[1 M]} \overline{\mathbf{V}}_{\mathbf{a}} \\
\vdots & \vdots & \ddots & \vdots \\
\tilde{\mathbf{H}}_{\frac{M}{2} a}^{[11]} \overline{\mathbf{V}}_{\mathbf{a}} & \tilde{\mathbf{H}}_{\frac{M}{2} a}^{[12]} \overline{\mathbf{V}}_{\mathbf{a}} & \cdots & \tilde{\mathbf{H}}_{\frac{M}{2} a}^{[1 M]} \overline{\mathbf{V}}_{\mathbf{a}} \\
\tilde{\mathbf{H}}_{\left(\frac{M}{2}+1\right) b}^{[11]} \overline{\mathbf{V}}_{\mathbf{b}} & \tilde{\mathbf{H}}_{\left(\frac{M}{2}+1\right) b}^{[12]} \overline{\mathbf{V}}_{\mathbf{b}} & \cdots & \tilde{\mathbf{H}}_{\left(\frac{M}{2}+1\right) b}^{[1 M]} \overline{\mathbf{V}}_{\mathbf{b}} \\
\vdots & \vdots & \ddots & \vdots \\
\tilde{\mathbf{H}}_{M b}^{[11]} \overline{\mathbf{V}}_{\mathbf{b}} & \tilde{\mathbf{H}}_{M b}^{[12]} \overline{\mathbf{V}}_{\mathbf{b}} & \cdots & \tilde{\mathbf{H}}_{M b}^{[1 M]} \overline{\mathbf{V}}_{\mathbf{b}}
\end{array}\right]
$$

wherein $\overline{\mathbf{H}}_{m a}^{[1 i]}$ is a diagonal square matrix of dimension $|\overline{\mathbf{V}}| \times|\overline{\mathbf{V}}|$ obtained from the first $|\overline{\mathbf{V}}|$ rows and columns of matrix $\mathbf{H}_{m}^{[1 i]}, \overline{\mathbf{H}}_{m b}^{[1 i]}$ is a diagonal square matrix of dimension $|\overline{\mathbf{V}}| \times|\overline{\mathbf{V}}|$ obtained from the last $|\overline{\mathbf{V}}|$ rows and columns of matrix $\mathbf{H}_{m}^{[1 i]} . \overline{\mathbf{V}}_{\mathbf{a}}$ is the $|\overline{\mathbf{V}}| \times|\overline{\mathbf{V}}|$ matrix obtained from the first $|\overline{\mathbf{V}}|$ rows of matrix $\overline{\mathbf{V}}$ and $\overline{\mathbf{V}}_{\mathbf{b}}$ is the $|\overline{\mathbf{V}}| \times|\overline{\mathbf{V}}|$ matrix obtained from the last $|\overline{\mathbf{V}}|$ rows of matrix $\overline{\mathbf{V}}$. Note that $\overline{\mathbf{D}}^{[1]}$ has $M|\overline{\mathbf{V}}|$ rows corresponding to $M$ receiver antennas and $M|\overline{\mathbf{V}}|$ columns corresponding to the desired signals from $M$ transmitters.

We will prove that $\operatorname{det}\left(\overline{\mathbf{D}}^{[1]}\right) \neq 0$ almost surely. The determinant of the matrix $\overline{\mathbf{D}}^{[1]}$ is a polynomial function of its entries. This polynomial is either identically a zero polynomial i.e., zero for all realizations, such as $x-x$, or it is not identically a zero polynomial, i.e., there exist some realizations for which the polynomial takes non-zero values. If a polynomial is not identically a zero polynomial, then it is not equal to zero almost surely for randomly generated channel coefficients, see e.g., the Schwartz Zippel Lemma [12], [30], [31]. Therefore, in order to show that a polynomial is almost surely non-zero for random realizations it suffices to show that it is not identically a zero-polynomial, i.e., that it is non-zero for at least one realization. So we show that the polynomial is not a zero polynomial by finding one specific set of channel coefficients such that the polynomial is not equal to zero.

We will set the channel coefficients such that $\overline{\mathbf{D}}^{[1]}$ becomes a block diagonal matrix with $M$ full rank blocks, which implies that $\overline{\mathbf{D}}^{[1]}$ is full rank, almost surely.

$$
\overline{\mathbf{D}}^{[1]}=\left[\begin{array}{ccccc}
\tilde{\mathbf{H}}_{1 a}^{[11]} \overline{\mathbf{V}}_{\mathbf{a}} \cdots & 0 & \cdots & 0 \\
\vdots & \ddots & \vdots & \ddots & \vdots \\
0 & \cdots \tilde{\mathbf{H}}_{\frac{M}{2} a}^{\left[1 \frac{M}{2}\right]} \overline{\mathbf{V}}_{\mathbf{a}} \cdots & 0 \\
\vdots & \ddots & \vdots & \ddots & \vdots \\
0 & \cdots & 0 & \cdots \tilde{\mathbf{H}}_{M b}^{[1 M]} \overline{\mathbf{V}}_{\mathbf{b}}
\end{array}\right]
$$

$\overline{\mathbf{D}}^{[1]}$ corresponds to the desired signal from transmitters $1, \cdots, M$ to Receiver 1 . We set all rows except rows $(i-1)|\overline{\mathbf{V}}|+1, \cdots, i|\overline{\mathbf{V}}|$ of $\overline{\mathbf{D}}^{[1]}$ to zero by choosing corresponding channel coefficients in the matrices $\overline{\mathbf{H}}_{m a}^{[1 k]}, \overline{\mathbf{H}}_{m b}^{[1 k]}, m \neq k$ to zero. Choosing these channel coefficients to be zero does not violate the rank constraints in the original network since $D_{0} \geq \frac{M}{2}$. It is for this reason that we choose top $|\overline{\mathbf{V}}|$ rows for first $\frac{M}{2}$ antennas and last $|\overline{\mathbf{V}}|$ rows for last $\frac{M}{2}$ antennas, corresponding to different timeslots. Note that this can be done because $\overline{\mathbf{V}}$ does not contain the desired channel coefficients associated with Receiver 1 . We have converted $\overline{\mathbf{D}}^{[1]}$ into a block diagonal matrix wherein each block is of size $|\overline{\mathbf{V}}| \times|\overline{\mathbf{V}}|$. We now show that each block is full rank almost surely. From the construction of $\overline{\mathbf{V}}_{a}$ and $\overline{\mathbf{V}}_{b}$, note that they are full rank matrices since linearly dependent columns have been discarded in $\overline{\mathbf{V}}$, as in Section VI-A, and various rows correspond to cross channel coefficients of different timeslots. Further, since $\overline{\mathbf{H}}_{m}^{[1 i]}, m \in\{1, \cdots, M\}$ are all full rank diagonal matrices with elements independent of $\overline{\mathbf{V}}_{a}$ and $\overline{\mathbf{V}}_{b}$, and so, each block matrix is full rank. Therefore, the desired signal matrix $\overline{\mathbf{D}}^{[1]}$ is full rank almost surely. Similarly, it can be shown that desired signal matrices $\overline{\mathbf{D}}^{[k]}$ are full rank, almost surely, at other receivers $k \in\{2, \ldots, K\}$.

Step 2: We will now prove that the interference space does not overlap with the desired signal space at the receivers. To this end, we first reorder the rows of matrix $\mathbf{F}^{[1]}=$ $\left[\mathbf{D}^{[1]} \mathbf{E}^{[1]}\right]$, arranging them according to the channel use slots. Desired signal received at channel index $\kappa$ is given by

$$
\mathbf{D}^{[1]}(\kappa)=\left[\begin{array}{ccc}
H_{1}^{[11]}(\kappa) & \cdots & H_{1}^{[1 M]}(\kappa) \\
H_{2}^{[11]}(\kappa) & \cdots & H_{2}^{[1 M]}(\kappa) \\
\vdots & \ddots & \vdots \\
H_{M}^{[11]}(\kappa) & \cdots & H_{M}^{[1 M]}(\kappa)
\end{array}\right] \otimes \overline{\mathbf{V}}(\kappa,:)
$$

At Receiver 1, interference caused by signals intended for receivers $j=2, \ldots, K$ at channel index $\kappa$, is given as

$$
\mathbf{E}^{[1]}(\kappa)=\mathbf{I}_{M} \otimes \overline{\mathcal{I}}(\kappa,:)
$$

As a result, signals received at channel use index $\kappa$ is

$$
\mathbf{F}^{[1]}(\kappa)=\left[\mathbf{D}^{[1]}(\kappa) \mathbf{E}^{[1]}(\kappa)\right]
$$

After rearranging the rows of $\mathbf{F}^{[1]}$ as above, it can be written as

$$
\mathbf{F}^{[1]}=\left[\begin{array}{c}
\mathbf{F}^{[1]}(1) \\
\vdots \\
\mathbf{F}^{[1]}(n)
\end{array}\right]
$$

We now describe the proofs for the matrix $\mathbf{F}^{[1]}$ containing desired and interference signals being full rank, first for $M=2$, followed by that for arbitrary $M$.

i) Linear independence proof for $M=2$ : Consider the signal space at Receiver 1, represented by matrix $\mathbf{F}^{[1]}$ of size $2 n \times 2 n$, wherein $n=|\overline{\mathbf{V}}|+|\overline{\mathcal{I}}|$. In this matrix, the first $2|\overline{\mathbf{V}}|$ columns correspond to the desired signal, and the last $2|\overline{\mathcal{I}}|$ columns correspond to the interference signal.

$$
\mathbf{F}^{[1]}=\left[\mathbf{D}^{[1]} \mathbf{E}^{[1]}\right]_{2 n \times 2 n}
$$




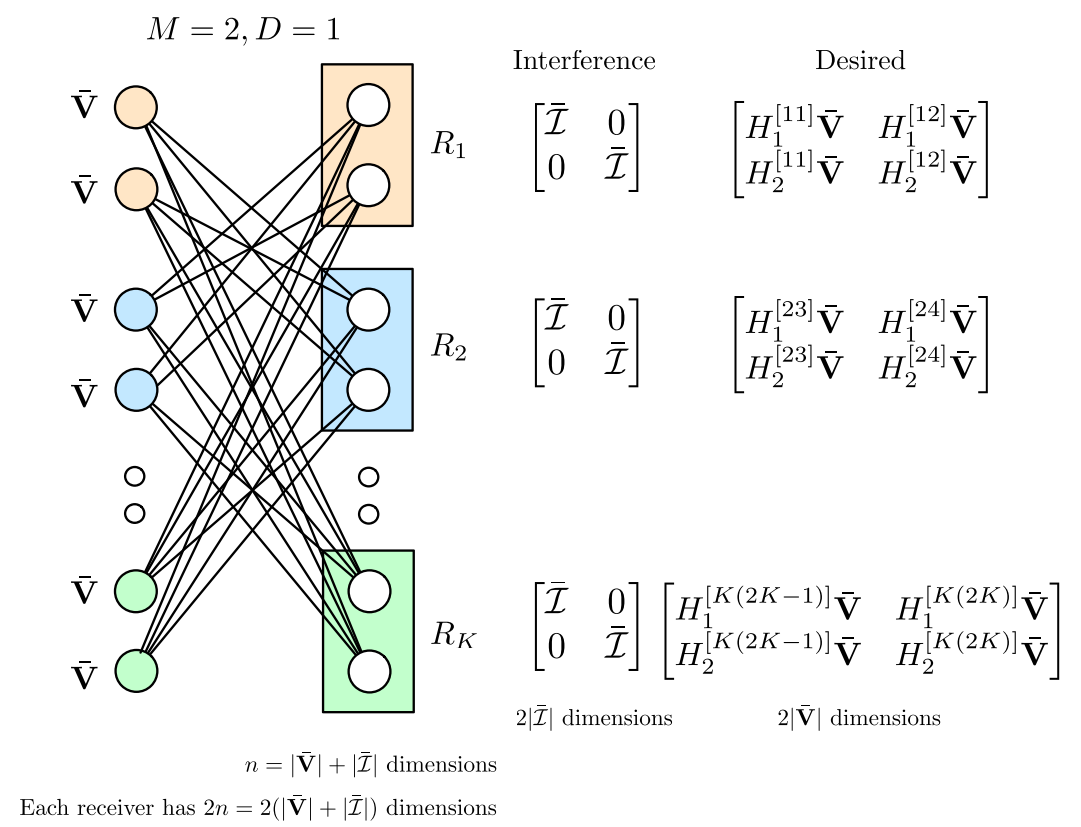

Fig. 12. Asymptotic alignment for $K$-user rank deficient channel with $M=2$.

wherein the columns corresponding to the desired signals are represented using $\mathbf{D}^{[1]}$

$$
\begin{aligned}
\mathbf{D}^{[1]} & =\left[\begin{array}{c}
\mathbf{D}^{[1]}(1) \\
\mathbf{D}^{[1]}(2) \\
\vdots \\
\mathbf{D}^{[1]}(n)
\end{array}\right]_{2 n \times 2|\overline{\mathbf{V}}|} \\
\mathbf{D}^{[1]}(\kappa) & =\mathbf{H}_{11}(\kappa) \otimes \overline{\mathbf{V}}(\kappa,:) \\
& =\left[\begin{array}{cc}
\overline{\mathbf{H}}_{1}^{[1]]}(\kappa) \overline{\mathbf{V}}(\kappa,:) & \overline{\mathbf{H}}_{1}^{[12]}(\kappa) \overline{\mathbf{V}}(\kappa,:) \\
\overline{\mathbf{H}}_{2}^{[11]}(\kappa) \overline{\mathbf{V}}(\kappa,:) & \overline{\mathbf{H}}_{2}^{[12]}(\kappa) \overline{\mathbf{V}}(\kappa,:)
\end{array}\right]_{2 \times 2|\overline{\mathbf{V}}|}
\end{aligned}
$$

and the columns corresponding to the interference signals are represented using $\mathbf{E}^{[1]}$ of size $2 n \times 2|\overline{\mathcal{I}}|$

$\mathbf{E}^{[1]}=\left[\begin{array}{cccccc}\overline{\mathcal{I}}(1,1) & \cdots & \overline{\mathcal{I}}(1,|\overline{\mathcal{I}}|) & 0 & \cdots & 0 \\ 0 & \cdots & 0 & \overline{\mathcal{I}}(1,1) & \cdots & \overline{\mathcal{I}}(1,|\overline{\mathcal{I}}|) \\ \overline{\mathcal{I}}(2,1) & \cdots & \overline{\mathcal{I}}(2,|\overline{\mathcal{I}}|) & 0 & \cdots & 0 \\ 0 & \cdots & 0 & \overline{\mathcal{I}}(2,1) & \cdots & \overline{\mathcal{I}}(2,|\overline{\mathcal{I}}|) \\ \vdots & \ddots & \vdots & \vdots & \vdots & \vdots \\ \overline{\mathcal{I}}(|\overline{\mathbf{V}}|, 1) & \cdots & \overline{\mathcal{I}}(|\overline{\mathbf{V}}|,|\overline{\mathcal{I}}|) & 0 & \cdots & 0 \\ 0 & \cdots & 0 & \overline{\mathcal{I}}(|\overline{\mathbf{V}}|, 1) & \cdots & \overline{\mathcal{I}}(|\overline{\mathbf{V}}|,|\overline{\mathcal{I}}|) \\ \vdots & \ddots & \vdots & \vdots & \vdots & \vdots \\ \overline{\mathcal{I}}(n, 1) & \cdots & \overline{\mathcal{I}}(n,|\overline{\mathcal{I}}|) & 0 & \cdots & 0 \\ 0 & \cdots & 0 & \overline{\mathcal{I}}(n, 1) & \cdots & \overline{\mathcal{I}}(n,|\overline{\mathcal{I}}|)\end{array}\right]$

where $\overline{\mathbf{V}}(\kappa,:)$ denotes $\kappa$-th row of $\overline{\mathbf{V}}$, and $\overline{\mathcal{I}}(k, l)$ denotes the element in the $k$-th row and $l$-th column of $\overline{\mathcal{I}}$. Note that 2 consecutive rows correspond to same timeslot, and signals correspond to $n$ timeslots.

Suppose the $2 \times 2$ direct channel between Transmitter 1 and Receiver 1 is of rank 1 (as shown in Fig 12), then without loss of generality, the desired signal matrix corresponding to channel use index $\kappa, \mathbf{D}^{[1]}(\kappa)$ can be written as

$$
\mathbf{D}^{[1]}(\kappa)=\left[\begin{array}{cc}
\overline{\mathbf{H}}_{1}^{[11]}(\kappa) \overline{\mathbf{V}}(\kappa,:) & \overline{\mathbf{H}}_{1}^{[12]}(\kappa) \overline{\mathbf{V}}(\kappa,:) \\
\alpha_{\kappa} \overline{\mathbf{H}}_{1}^{[11]}(\kappa) \overline{\mathbf{V}}(\kappa,:) & \alpha_{\kappa} \overline{\mathbf{H}}_{1}^{[12]}(\kappa) \overline{\mathbf{V}}(\kappa,:)
\end{array}\right]_{2 \times 2|\overline{\mathbf{V}}|}
$$

We will now show that the determinant of matrix $\mathbf{F}^{[1]}$ has a unique monomial which implies that $\operatorname{det}\left(\mathbf{F}^{[1]}\right) \neq 0$, almost surely. Expanding the determinant of $\mathbf{F}^{[1]}$ along the interference signal columns corresponding to $\mathbf{E}^{[1]}$, it can be noted that $\operatorname{det}\left(\mathbf{F}^{[1]}\right)$ contains the polynomial $\mathcal{I}_{P} \operatorname{det}(\mathbf{X})$ with $\mathcal{I}_{P}=\mathcal{I}_{A} \mathcal{I}_{B}$ and

$$
\begin{aligned}
& \mathcal{I}_{A}=\left(\prod_{i=1}^{|\overline{\mathbf{V}}|} \overline{\mathcal{I}}_{i}(i)\right) \times\left(\prod_{i=|\overline{\mathbf{V}}|+1}^{|\overline{\mathcal{I}}|} \overline{\mathcal{I}}_{i-|\overline{\mathbf{V}}|}(i)\right) \\
& \mathcal{I}_{B}=\prod_{i=|\overline{\mathbf{V}}|+1}^{n} \overline{\mathcal{I}}_{i-|\overline{\mathbf{V}}|}(i)
\end{aligned}
$$

wherein $\mathcal{I}_{A}$ is the product of interference terms from even rows of $\mathbf{F}^{[1]}, \mathcal{I}_{B}$ is the product of interference terms from odd rows of $\mathbf{F}^{[1]}, \mathcal{I}_{P}=\mathcal{I}_{A} \mathcal{I}_{B}$ represents the product of interference terms from the columns of $\mathbf{E}^{[1]}$ described above, and the matrix $\mathbf{X}$ is of size $2|\overline{\mathbf{V}}| \times 2|\overline{\mathbf{V}}|$, given as

$$
\mathbf{X}=\left[\begin{array}{cc}
\alpha_{1} \overline{\mathbf{H}}_{1}^{[11]}(1) \overline{\mathbf{V}}(1,:) & \alpha_{1} \overline{\mathbf{H}}_{1}^{[12]}(1) \overline{\mathbf{V}}(1,:) \\
\alpha_{2} \overline{\mathbf{H}}_{1}^{[11]}(2) \overline{\mathbf{V}}(2,:) & \alpha_{2} \overline{\mathbf{H}}_{1}^{[12]}(2) \overline{\mathbf{V}}(2,:) \\
\vdots & \vdots \\
\alpha_{1} \overline{\mathbf{H}}_{1}^{[11]}(|\overline{\mathbf{V}}|) \overline{\mathbf{V}}(|\overline{\mathbf{V}}|,:) & \alpha_{1} \overline{\mathbf{H}}_{1}^{[12]}(|\overline{\mathbf{V}}|) \overline{\mathbf{V}}(|\overline{\mathbf{V}}|,:) \\
\overline{\mathbf{H}}_{1}^{[1]}(|\overline{\mathcal{I}}|+1) \overline{\mathbf{V}}(|\overline{\mathcal{I}}|+1,:) \overline{\mathbf{H}}_{1}^{[12]}(|\overline{\mathcal{I}}|+1) \overline{\mathbf{V}}(|\overline{\mathcal{I}}|+1,:) \\
\vdots & \vdots \\
\overline{\mathbf{H}}_{1}^{[11]}(n) \overline{\mathbf{V}}(n,:) & \overline{\mathbf{H}}_{1}^{[12]}(n) \overline{\mathbf{V}}(n,:)
\end{array}\right]
$$


and $\operatorname{det}(\mathbf{X})=\left(\prod_{i=1}^{|\overline{\mathbf{V}}|} \alpha_{i}\right) \operatorname{det}(\overline{\mathbf{X}})$

wherein the matrix $\overline{\mathbf{X}}$ is of size $2|\overline{\mathbf{V}}| \times 2|\overline{\mathbf{V}}|$, given as

$$
\overline{\mathbf{X}}=\left[\begin{array}{cc}
\overline{\mathbf{H}}_{1}^{[11]}(1) \overline{\mathbf{V}}(1,:) & \overline{\mathbf{H}}_{1}^{[12]}(1) \overline{\mathbf{V}}(1,:) \\
\overline{\mathbf{H}}_{1}^{[11]}(2) \overline{\mathbf{V}}(2,:) & \overline{\mathbf{H}}_{1}^{[12]}(2) \overline{\mathbf{V}}(2,:) \\
\vdots & \vdots \\
\overline{\mathbf{H}}_{1}^{[11]}(|\overline{\mathbf{V}}|) \overline{\mathbf{V}}(|\overline{\mathbf{V}}|,:) & \overline{\mathbf{H}}_{1}^{[12]}(|\overline{\mathbf{V}}|) \overline{\mathbf{V}}(|\overline{\mathbf{V}}|,:) \\
\overline{\mathbf{H}}_{1}^{[11]}(|\overline{\mathcal{I}}|+1) \overline{\mathbf{V}}(|\overline{\mathcal{I}}|+1,:) & \overline{\mathbf{H}}_{1}^{[12]}(|\overline{\mathcal{I}}|+1) \overline{\mathbf{V}}(|\overline{\mathcal{I}}|+1,:) \\
\vdots & \vdots \\
\overline{\mathbf{H}}_{1}^{[11]}(n) \overline{\mathbf{V}}(n,:) & \overline{\mathbf{H}}_{1}^{[12]}(n) \overline{\mathbf{V}}(n,:)
\end{array}\right]
$$

Different choice for interference terms in $\mathcal{I}_{P}\left(\mathcal{I}_{P} \neq\right.$ $\mathcal{I}_{A} \mathcal{I}_{B}$ ), result in either a different matrix $\tilde{\mathbf{X}}$ (instead of $\mathbf{X}$ ) corresponding to rows from different timeslots, or another distinct product of $\alpha_{i}$ in the determinant expression of same $\mathbf{X}$, instead of that in (75). Choosing a different row for each term $\overline{\mathcal{I}}_{k}(\kappa)$ than from one above, results in either a different matrix $\mathbf{X}$ or a different product of $\alpha_{i}$ coefficients. Note that $\operatorname{det}(\mathbf{X})$ is a non-zero polynomial since rows correspond to different timeslots, and elements can be chosen such that one instance of the determinant polynomial is non-zero. Also, each element of matrix $\mathbf{X}$ has direct channels which are not present in all elements of $\overline{\mathcal{I}}$. Elements of $\overline{\mathcal{I}}$ are distinct powers of the cross channels with non-zero entries. Thus, we have a unique non-zero polynomial $\mathcal{I}_{A} \mathcal{I}_{B} \operatorname{det}(\mathbf{X})$ in the determinant expression of $\mathbf{F}^{[1]}$ and so the determinant of $\mathbf{F}^{[1]}$ is non-zero, almost surely. Similarly, we can show that all matrices $\mathbf{F}^{[k]}, k \in\{2, \ldots, K\}$ are full rank, corresponding to signal space at different receivers. Suppose the $2 \times 2$ direct channel between Transmitter 1 and Receiver 1 is full rank, matrix $\mathbf{F}^{[1]}$ can be similarly shown to be full rank, almost surely.

Thus the desired signal is linearly independent from the interference at each receiver and therefore, the total accessible DoF for Receiver $j$ equals $M \frac{2|\overline{\mathbf{V}}|}{2 n}=M \frac{2|\overline{\mathbf{V}}|}{2|\overline{\mathbf{V}}|+2|\overline{\mathcal{I}}|} \rightarrow \frac{M}{2}$ as $n \rightarrow \infty$, resulting in DoF of $\frac{M}{2}$ per user, as desired.

ii) Linear independence proof for arbitrary $\mathbf{M}$ : For arbitrary $M$, signal space containing desired signal and interference can be represented as:

$$
\mathbf{F}^{[1]}=\left[\mathbf{D}^{[1]} \mathbf{E}^{[1]}\right]_{n M \times n M}
$$

wherein the columns corresponding to the desired signals are represented using $\mathbf{D}^{[1]}$

$$
\begin{aligned}
\mathbf{D}^{[1]} & =\left[\begin{array}{c}
\mathbf{D}^{[1]}(1) \\
\mathbf{D}^{[1]}(2) \\
\vdots \\
\mathbf{D}^{[1]}(n)
\end{array}\right]_{n M \times M|\overline{\mathbf{V}}|} \\
\mathbf{D}^{[1]}(\kappa) & =\mathbf{H}_{11}(\kappa) \otimes \mathbf{\mathbf { V }}(\kappa,:)
\end{aligned}
$$

$$
=\left[\begin{array}{ccc}
\overline{\mathbf{H}}_{1}^{[11]}(\kappa) \overline{\mathbf{V}}(\kappa,:) & \cdots & \overline{\mathbf{H}}_{1}^{[1 M]}(\kappa) \overline{\mathbf{V}}(\kappa,:) \\
\overline{\mathbf{H}}_{2}^{[11]}(\kappa) \overline{\mathbf{V}}(\kappa,:) & \cdots & \overline{\mathbf{H}}_{2}^{[1 M]}(\kappa) \overline{\mathbf{V}}(\kappa,:) \\
\vdots & \ddots & \vdots \\
\overline{\mathbf{H}}_{M}^{[11]}(\kappa) \overline{\mathbf{V}}(\kappa,:) & \cdots & \overline{\mathbf{H}}_{M}^{[1 M]}(\kappa) \overline{\mathbf{V}}(\kappa,:)
\end{array}\right]
$$

and the columns corresponding to the interference signals are represented using $\mathbf{E}^{[1]}$, matrix shown in (79), as shown at the top of the next page, wherein $\overline{\mathbf{V}}(\kappa,:)$ denotes $\kappa$-th row of $\overline{\mathbf{V}}$, and $\overline{\mathcal{I}}(k, l)$ denotes the element in the $k$-th row and $l$-th column of $\overline{\mathcal{I}}$. When desired channels are of rank $D_{0}>\frac{M}{2}$, above matrix can be written as

$$
\begin{aligned}
\mathbf{D}^{[1]}(\kappa)= & \mathbf{H}_{11}(\kappa) \otimes \overline{\mathbf{V}}(\kappa,:) \\
= & {\left[\begin{array}{ccc}
\mathbf{a}_{1} & \mathbf{b}_{1} & \cdots \\
\vdots & \vdots & \vdots \\
\mathbf{a}_{\frac{M}{2}} & \mathbf{b}_{\frac{M}{2}} & \cdots \\
\sum_{i=1}^{\frac{M}{2}} \alpha_{i} a_{i} & \sum_{i=1}^{\frac{M}{2}} \alpha_{i} b_{i} & \cdots \\
\vdots & \vdots & \vdots \\
\sum_{i=1}^{\frac{M}{2}} \beta_{i} a_{i} & \sum_{i=1}^{\frac{M}{2}} \beta_{i} b_{i} & \cdots
\end{array}\right]_{M \times M|\overline{\mathbf{V}}|} }
\end{aligned}
$$

Expanding the determinant of $\mathbf{F}^{[1]}$ along the columns carrying interference, note that the determinant contains the polynomial $\mathcal{I}_{P} \operatorname{det}(\mathbf{X})$ with $\mathcal{I}_{P}=\mathcal{I}_{A} \mathcal{I}_{B}$ and

$$
\begin{aligned}
& \mathcal{I}_{A}=\left(\prod_{i=1}^{|\overline{\mathbf{V}}|} \overline{\mathcal{I}}_{i}^{\frac{M}{2}}(i)\right) \times\left(\prod_{i=|\overline{\mathbf{V}}|+1}^{|\overline{\mathcal{I}}|} \overline{\mathcal{I}}_{i-|\overline{\mathbf{V}}|}^{\frac{M}{2}}(i)\right) \\
& \mathcal{I}_{B}=\prod_{i=|\overline{\mathbf{V}}|+1}^{n} \overline{\mathcal{I}}_{i-|\overline{\mathbf{V}}|}^{\frac{M}{2}}(i)
\end{aligned}
$$

wherein $\mathcal{I}_{A}$ is the product of interference terms from the middle $\frac{M}{2}$ rows of each $M$-row matrix corresponding to same channel use, in $\mathbf{F}^{[1]}, \mathcal{I}_{B}$ is the product of interference terms from the top $\left\lceil\frac{M}{4}\right\rceil$ and the bottom $\left\lfloor\frac{M}{4}\right\rfloor$ rows of each $M$-row matrix corresponding to same channel use, in $\mathbf{F}^{[1]}$, and the matrix $\mathbf{X}$ is given as:

$$
\begin{aligned}
& \mathbf{X}=\left[\begin{array}{c}
\tilde{\mathbf{X}}(1) \\
\tilde{\mathbf{X}}(2) \\
\vdots \\
\tilde{\mathbf{X}}(|\overline{\mathbf{V}}|) \\
\mathbf{X}^{\prime}(|\overline{\mathcal{I}}|+1) \\
\vdots \\
\mathbf{X}^{\prime}(n)
\end{array}\right]_{M|\overline{\mathbf{V}}| \times M|\overline{\mathbf{V}}|} \\
& \tilde{\mathbf{X}}(k)=\left[\begin{array}{ccc}
\overline{\mathbf{H}}_{1}^{[11]}(k) \overline{\mathbf{V}}(k,:) & \cdots & \overline{\mathbf{H}}_{1}^{[1 M]}(k) \overline{\mathbf{V}}(k,:) \\
\vdots & \ddots & \vdots \\
\overline{\mathbf{H}}_{\left[\left\lceil\frac{M}{4}\right]\right.}^{[11]}(k) \overline{\mathbf{V}}(k,:) & \cdots & \overline{\mathbf{H}}_{\left\lceil\frac{M}{4}\right]}^{[1 M]}(k) \overline{\mathbf{V}}(k,:) \\
\overline{\mathbf{H}}_{\left\lceil\frac{3 M}{4}\right]}^{[11]]}(k) \overline{\mathbf{V}}(k,:) & \cdots & \overline{\mathbf{H}}_{\left[\frac{3 M}{4}\right]}^{[1 M]}(k) \overline{\mathbf{V}}(k,:) \\
\vdots & \ddots & \vdots \\
\overline{\mathbf{H}}_{M}^{[11]}(k) \overline{\mathbf{V}}(k,:) & \cdots & \overline{\mathbf{H}}_{M}^{[1 M]}(k) \overline{\mathbf{V}}(k,:)
\end{array}\right]
\end{aligned}
$$




$\mathbf{E}^{[1]}=\left[\begin{array}{cccccccccccc}\overline{\mathcal{I}}(1,1) & \cdots & \overline{\mathcal{I}}(1,|\overline{\mathcal{I}}|) & 0 & \cdots & 0 & 0 & \cdots & 0 & 0 & \cdots & 0 \\ 0 & \cdots & 0 & \overline{\mathcal{I}}(1,1) & \cdots & \overline{\mathcal{I}}(1,|\overline{\mathcal{I}}|) & 0 & \cdots & 0 & 0 & \cdots & 0 \\ \vdots & \vdots & \vdots & \vdots & \vdots & \vdots & \vdots & \ddots & \vdots & \vdots & \vdots & \vdots \\ 0 & \cdots & 0 & 0 & \cdots & 0 & 0 & \cdots & 0 & \overline{\mathcal{I}}(1,1) & \cdots & \overline{\mathcal{I}}(1,|\overline{\mathcal{I}}|) \\ \overline{\mathcal{I}}(2,1) & \cdots & \overline{\mathcal{I}}(2,|\overline{\mathcal{I}}|) & 0 & \cdots & 0 & 0 & \cdots & 0 & 0 & \cdots & 0 \\ 0 & \cdots & 0 & \overline{\mathcal{I}}(2,1) & \cdots & \overline{\mathcal{I}}(2,|\overline{\mathcal{I}}|) & 0 & \cdots & 0 & 0 & \cdots & 0 \\ \vdots & \vdots & \vdots & \vdots & \vdots & \vdots & \vdots & \ddots & \vdots & \vdots & \vdots & \vdots \\ 0 & \cdots & 0 & 0 & \cdots & 0 & 0 & \cdots & 0 & \overline{\mathcal{I}}(2,1) & \cdots & \overline{\mathcal{I}}(2,|\overline{\mathcal{I}}|) \\ \vdots & \vdots & \vdots & \vdots & \vdots & \vdots & \vdots & \ddots & \vdots & \vdots & \vdots & \vdots \\ \overline{\mathcal{I}}(|\mathbf{\mathbf { V }}|, 1) & \cdots & \overline{\mathcal{I}}(|\overline{\mathbf{V}}|,|\overline{\mathcal{I}}|) & 0 & \cdots & 0 & 0 & \cdots & 0 & 0 & \cdots & 0 \\ 0 & \cdots & 0 & \overline{\mathcal{I}}(|\overline{\mathbf{V}}|, 1) & \cdots & \overline{\mathcal{I}}(|\overline{\mathbf{V}}|,|\overline{\mathcal{I}}|) & 0 & \cdots & 0 & 0 & \cdots & 0 \\ \vdots & \vdots & \vdots & \vdots & \vdots & \vdots & \vdots & \ddots & \vdots & \vdots & \vdots & \vdots \\ 0 & \cdots & 0 & 0 & \cdots & 0 & 0 & \cdots & 0 & \overline{\mathcal{I}}(|\overline{\mathbf{V}}|, 1) & \cdots & \overline{\mathcal{I}}(|\overline{\mathbf{V}}|,|\overline{\mathcal{I}}|) \\ \vdots & \vdots & \vdots & \vdots & \vdots & \vdots & \vdots & \ddots & \vdots & \vdots & \vdots & \vdots \\ \overline{\mathcal{I}}(n, 1) & \cdots & \overline{\mathcal{I}}(n,|\overline{\mathcal{I}}|) & 0 & \cdots & 0 & 0 & \cdots & 0 & 0 & \cdots & 0 \\ 0 & \cdots & 0 & \overline{\mathcal{I}}(n, 1) & \cdots & \overline{\mathcal{I}}(n,|\overline{\mathcal{I}}|) & 0 & \cdots & 0 & 0 & \cdots & 0 \\ \vdots & \vdots & \vdots & \vdots & \vdots & \vdots & \vdots & \ddots & \vdots & \vdots & \vdots & \vdots \\ 0 & \cdots & 0 & 0 & \cdots & 0 & 0 & \cdots & 0 & \overline{\mathcal{I}}(n, 1) & \cdots & \overline{\mathcal{I}}(n,|\overline{\mathcal{I}}|)\end{array}\right]_{n M \times M|\overline{\mathcal{I}}|}$

$$
\mathbf{X}^{\prime}(k)=\left[\begin{array}{cccc}
\overline{\mathbf{H}}_{\left\lceil\frac{M}{4}\right\rceil+1}^{[11]}(k) \overline{\mathbf{V}}(k,:) & \cdots & \overline{\mathbf{H}}_{\left\lceil\frac{M}{4}\right\rceil+1}^{[1 M]}(k) \overline{\mathbf{V}}(k,:) \\
\vdots & \ddots & \vdots \\
\overline{\mathbf{H}}_{\left\lceil\frac{3 M}{4}\right\rceil-1}^{[11]}(k) \overline{\mathbf{V}}(k,:) & \cdots & \overline{\mathbf{H}}_{\left\lceil\frac{3 M}{4}\right\rceil-1}^{[1 M]}(k) \overline{\mathbf{V}}(k,:)
\end{array}\right]
$$

When determinant of $\mathbf{X}$ is evaluated, we get terms of the form $a_{k}\left(\sum_{i=1}^{D_{0}} \beta_{i} b_{i}\right), k \in\left\{1, \cdots, D_{0}\right\}$ when $D_{0}>\frac{M}{2}$ corresponding to channel use $\kappa$, by considering (81). For obtaining same product of interference terms in $\mathcal{I}_{A}$ and $\mathcal{I}_{B}$ by choosing different rows, different linear combinations of $a_{i}, b_{i}, i \in\left\{1, \ldots, D_{0}\right\}$ are involved. Choosing a different row for each term $\overline{\mathcal{I}}_{k}(\kappa)$ in $\mathcal{I}_{P}$ than from one above, results in either a different matrix $\mathbf{X}$ or a different linear combination of $a_{i}, b_{i}, i \in\left\{1, \ldots, D_{0}\right\}$. Also, each element of matrix $\mathbf{X}$ has direct channels which are not present in all elements of $\overline{\mathcal{I}}$. Thus we have a unique non-zero polynomial $\mathcal{I}_{A} \mathcal{I}_{B} \operatorname{det}(\mathbf{X})$ in the determinant of $\mathbf{F}^{[1]}$, since any other choices for interference terms cannot result in the same polynomial, and so, $\operatorname{det}\left(\mathbf{F}^{[1]}\right) \neq 0$, almost surely. Similarly, we can show that all matrices $\mathbf{F}^{[k]}, k \in\{2, \ldots, K\}$ are full rank, corresponding to signal space at different receivers.

Hence the matrix $\mathbf{F}^{[k]}$ is full rank and we have proved the linear independence of desired and interfering signals, for all $M$. This implies that for the region $(K-1) D>M$, achievable DoF per user are $\min \left(D_{0}, \frac{M}{2}\right)$.

\section{Theorem 3: Proof of Outer Bound}

We first prove that the DoF outer bound per user for region $(K-1) D \leq M$ is given by $M-\frac{(K-1) D}{2}$, and then prove that $\frac{M}{2}$ is the DoF outer bound per user for regions $(K-2) D \leq M<(K-1) D$ and $(K-2) D>M$.

\section{C.1) K-User Channel With $(\mathbf{K}-\mathbf{1}) \mathbf{D} \leq \mathbf{M}$ :}

\section{C.1.a) Change of basis:}

Step 1: For Receiver $k$, we design a $M \times M$ square matrix $\mathbf{R}_{k}$. First, we determine $(K-1) D$ rows at Receiver $k$. The linear transformation is designed such that first $D$ antennas of Receiver $k$ hear interference only from Transmitter $k+1$, next $D$ antennas of Receiver $k$ hear interference only from Transmitter $k+2$, and so on for $K-1$ steps, where $D$ antennas of Receiver $k$ hear interference from only Transmitter $k+K-1$. This operation is guaranteed since $\operatorname{rank}\left(\mathbf{H}_{k(k+i)}\right)=D, i \neq 0$, and vectors can be chosen from the corresponding common nullspaces. For Receiver $k$, these are denoted as $S_{k a_{1}}, \cdots, S_{k a_{K-1}}$. Remaining $M-(K-1) D$ rows, denoted as $S_{k c}$, are chosen so that they do not hear any interference, which is also possible since the common nullspace of $K-1$ cross channels has $M-(K-1) D$ dimensions.

Step 2: Within the $M$-dimensional signal space at Transmitter $k$, there is $M-(K-1) D$ dimensional subspace orthogonal to $(K-1) D$ receiver antennas $(k-i) a_{i}, \forall i=\{1, \ldots, K-1\}$. These $K-1$ subspaces have $M-(K-1) D$ dimensional intersection as seen by Transmitter $k$. We will choose $M-(K-1) D$ columns of a $M \times M$ matrix $\mathbf{T}_{k}$ at Transmitter $k$, from this intersection. These will not be seen at any of unintended receivers, and are denoted as $X_{k c}$ at Transmitter $k$.

Step 3: Then, we choose other columns of $\mathbf{T}_{k}$ such that $D$ antennas of Transmitter $k$ are heard only by receiver antennas $(k-i) a_{i}, \forall i=\{1, \ldots, K-1\}$. This operation is guaranteed since $\operatorname{rank}\left(\mathbf{H}_{k(k+i)}\right)=D, i \neq 0$, and we can choose vectors from the corresponding common nullspaces. For Transmitter $k$, these are denoted as $X_{k a_{1}}, \cdots, X_{k a_{K-1}}$. The resulting network connectivity after the change of basis operations is shown in Figure 13. 


\begin{tabular}{r|r|r|}
\hline$\left|X_{1 a_{1}}\right|=D$ & $X_{1 a_{1}}$ & $\circ$ \\
\hline$\left|X_{1 a_{2}}\right|=D$ & $X_{1 a_{2}}$ & $\circ$ \\
\hline$\circ$ & $\circ$ & $\circ$ \\
\hline$\left|X_{1 a_{K-1}}\right|=D$ & $X_{1 a_{K-1}}$ & $\circ$ \\
\hline$\left|X_{1 c}\right|=M-(K-1) D \geq 0$ & $X_{1 c}$ & $\circ$ \\
\hline
\end{tabular}

\begin{tabular}{c|l|l}
\hline$\circ$ & $S_{1 a_{1}}\left(X_{2 a_{1}}\right)$ & $\left|S_{1 a_{1}}\right|=D$ \\
\hline$\circ$ & $S_{1 a_{2}}\left(X_{3 a_{2}}\right)$ & $\left|S_{1 a_{2}}\right|=D$ \\
\hline$\circ$ & $\circ$ & $\circ$ \\
\hline$\circ$ & $S_{1 a_{K-1}}\left(X_{K a_{K-1}}\right)$ & $\left|S_{1 a_{K-1}}\right|=D$ \\
\hline$\circ$ & $S_{1 c}()$ & $\left|S_{1 c}\right|=M-(K-1) D \geq 0$ \\
\hline
\end{tabular}

\begin{tabular}{r|r|c|}
\hline$\left|X_{2 a_{1}}\right|=D$ & $X_{2 a_{1}}$ & $\circ$ \\
\hline$\left|X_{2 a_{2}}\right|=D$ & $X_{2 a_{2}}$ & $\circ$ \\
\hline$\circ$ & $\circ$ & $\circ$ \\
\hline$\left|X_{2 a_{K-1}}\right|=D$ & $X_{2 a_{K-1}}$ & $\circ$ \\
\hline$\left|X_{2 c}\right|=M-(K-1) D \geq 0$ & $X_{2 c}$ & $\circ$ \\
\hline
\end{tabular}

\begin{tabular}{|l|l|l}
\hline$\circ$ & $S_{2 a_{1}}\left(X_{3 a_{1}}\right)$ & $\left|S_{2 a_{1}}\right|=D$ \\
\hline$\circ$ & $S_{2 a_{2}}\left(X_{4 a_{2}}\right)$ & $\left|S_{2 a_{2}}\right|=D$ \\
\hline$\circ$ & $\circ$ & $\circ$ \\
\hline$\circ$ & $S_{2 a_{K-1}}\left(X_{1 a_{K-1}}\right)$ & $\left|S_{2 a_{K-1}}\right|=D$ \\
\hline$\circ$ & $S_{2 c}()$ & $\left|S_{2 c}\right|=M-(K-1) D \geq 0$ \\
\hline
\end{tabular}

\begin{tabular}{r|r|r|}
\hline$\left|X_{3 a_{1}}\right|=D$ & $X_{3 a_{1}}$ & $\circ$ \\
\hline$\left|X_{3 a_{2}}\right|=D$ & $X_{3 a_{2}}$ & $\circ$ \\
\hline$\circ$ & $\circ$ & $\circ$ \\
\hline$\left|X_{3 a_{K-1}}\right|=D$ & $X_{3 a_{K-1}}$ & $\circ$ \\
\hline$\left|X_{3 c}\right|=M-(K-1) D \geq 0$ & $X_{3 c}$ & $\circ$ \\
\hline
\end{tabular}

\begin{tabular}{|l|l|l}
\hline$\circ$ & $S_{3 a_{1}}\left(X_{4 a_{1}}\right)$ & $\left|S_{3 a_{1}}\right|=D$ \\
\hline$\circ$ & $S_{3 a_{2}}\left(X_{5 a_{2}}\right)$ & $\left|S_{3 a_{2}}\right|=D$ \\
\hline$\circ$ & $\circ$ & $\circ$ \\
\hline$\circ$ & $S_{3 a_{K-1}}\left(X_{2 a_{K-1}}\right)$ & $\left|S_{3 a_{K-1}}\right|=D$ \\
\hline$\circ$ & $S_{3 c}()$ & $\left|S_{3 c}\right|=M-(K-1) D \geq 0$ \\
\hline$\circ$ & \multicolumn{2}{|l}{} \\
\hline
\end{tabular}

\begin{tabular}{r|r|r|}
\hline$\left|X_{K a_{1}}\right|=D$ & $X_{K a_{1}}$ & $\circ$ \\
\hline$\left|X_{K a_{2}}\right|=D$ & $X_{K a_{2}}$ & $\circ$ \\
\hline$\circ$ & $\circ$ & $\circ$ \\
\hline$\left|X_{K a_{K-1}}\right|=D$ & $X_{K a_{K-1}}$ & $\circ$ \\
\hline$\left|X_{K c}\right|=M-(K-1) D \geq 0$ & $X_{K c}$ & $\circ$ \\
\hline
\end{tabular}

$\circ$
\begin{tabular}{|l|l|l}
\hline$\circ$ & $S_{K a_{1}}\left(X_{1 a_{1}}\right)$ & $\left|S_{K a_{1}}\right|=D$ \\
\hline$\circ$ & $S_{K a_{2}}\left(X_{2 a_{2}}\right)$ & $\left|S_{K a_{2}}\right|=D$ \\
\hline$\circ$ & $\circ$ & $\circ$ \\
\hline$\circ$ & $S_{K a_{K-1}}\left(X_{(K-1) a_{K-1}}\right)$ & $\left|S_{K a_{K-1}}\right|=D$ \\
\hline$\circ$ & $S_{K c}()$ & $\left|S_{K c}\right|=M-(K-1) D \geq 0$ \\
\hline
\end{tabular}

Fig. 13. Outer bound: $K$-user rank deficient interference channel, $(K-1) D<M$.

C.1.b) Outer bound: Without loss of generality let us consider Receiver 1. As usual, for the outer bounds we will provide Receiver 1 enough side information through a genie so that it can decode all $K$ messages. The receiver can decode its desired message by assumption (because the given coding scheme is guaranteed to be reliable). After subtracting the signal from Transmitter 1, the receiver proceeds to decode the remaining $K-1$ messages. For the purpose, the genie information given to Receiver 1 is comprised of $(K-1)(M-D)$ dimensions $X_{2 a_{2}}^{n}, \ldots, X_{2 a_{K-1}}^{n}, X_{2 c}^{n}, X_{3 a_{1}}^{n}, X_{3 a_{3}}^{n}, \ldots, X_{3 a_{K-1}}^{n}, X_{3 c}^{n}, X_{K a_{1}}^{n}$, $\ldots, X_{K a_{K-2}}^{n}, X_{K c}^{n}$ which are not already heard by Receiver 1 . Then the total number of dimensions available to Receiver 1 (including those provided by the genie) is equal to:

$$
\begin{aligned}
(K-1) D+\left|\mathcal{G}_{1}\right| & =(K-1) D+(K-1)(M-D) \\
& =(K-1) M
\end{aligned}
$$

With these $(K-1) M$ dimensions, Receiver 1 will be able to resolve all $K-1$ interfering signals and can decode all $K$ messages. For example, $\mathcal{G}_{1}=\left\{X_{2 a_{2}}^{n}, X_{2 a_{3}}^{n}\right.$, $\left.X_{2 c}^{n}, X_{3 a_{1}}^{n}, X_{3 a_{3}}^{n}, X_{3 c}^{n}, X_{4 a_{1}}^{n}, X_{4 a_{2}}^{n}, X_{4 c}^{n}\right\}$ for $K=4$. Therefore, we have:

$$
\begin{aligned}
n R_{\Sigma} \leq & M n \log \rho+h\left(\mathcal{G}_{1} \mid \bar{Y}_{1}^{n}\right)+n o(\log \rho)+o(n) \\
\leq & M n \log \rho+h\left(X_{K a_{1}}^{n} \mid \bar{Y}_{1}^{n}\right)+\ldots+h\left(X_{K a_{K-2}}^{n} \mid \bar{Y}_{1}^{n}\right) \\
& +h\left(X_{K c}^{n} \mid \bar{Y}_{1}^{n}\right)+h\left(X_{2 a_{2}}^{n}, \ldots, X_{2 a_{K-1}}^{n}, X_{2 c}^{n} \mid \bar{Y}_{1}^{n}\right)
\end{aligned}
$$

where (87) follows from Fano's inequality and Lemma 1. (88) follows from applying chain rule and dropping some condition terms. (89) follows from the fact that dropping condition 
terms cannot decrease the differential entropy. Thus, we only keep $S_{1 a_{1}}^{n}, S_{1 a_{2}}^{n}, \ldots S_{1 a_{K-1}}^{n}$ as the condition terms which are $X_{2 a_{1}}^{n}, X_{3 a_{2}}^{n}, \ldots, X_{K a_{K-1}}^{n}$ respectively. (90) is obtained because from the observations of $\left(X_{k a_{1}}^{n}, X_{k a_{2}}^{n}, \ldots, X_{k a_{K-1}}^{n}, X_{k c}^{n}\right)$ we can decode $W_{k}, \forall k \in\{1, \ldots, K\}$ subject to the noise distortion, (91) follows since entropy of $X_{K c}^{n}$ is constrained by $M-(K-1) D$ antennas.

By advancing user indices considering all receivers, we have:

$$
\begin{aligned}
K n R_{\Sigma} \leq & (2 M-(K-1) D) n \log \rho+(K-2) n R_{\Sigma} \\
& +n o(\log \rho)+o(n) .
\end{aligned}
$$

which implies that the DoF per user are bounded above as

$$
d \leq M-\frac{(K-1) D}{2}
$$

C.2) K-User Channel With $(\mathbf{K}-\mathbf{2}) \mathbf{D} \leq \mathbf{M}<(\mathbf{K}-\mathbf{1}) \mathbf{D}$ : C.2.a) Change of basis:

Step 1: For Receiver $k$, we design a $M \times M$ square matrix $\mathbf{R}_{k}$. First, we determine the top $(K-2) D$ rows at Receiver $k$ in a way that the signals from transmitters $k+1, \cdots, k+K-2$ are separated from each other (signals from Transmitter $k+K-1$ remain in the mix). The linear transformation is designed such that the first $D$ antennas of Receiver $k$ hear only transmitters $k+1$ and $k+K-1$, next $D$ antennas of Receiver $k$ hear only transmitters $k+2$ and $k+K-1$, and so on such that at the $(K-2)^{t h}$ step we have $D$ antennas of Receiver $k$ that hear only transmitters $k+K-2$ and $k+K-1$. This operation is guaranteed since $M \geq(K-2) D$ and the subspaces seen by Receiver $k$ are in general position. The signals seen at Receiver $k$ by the first $K-2$ sets of $D$ antennas each are denoted as $S_{k a_{1}}, \cdots, S_{k a_{K-2}}$. Finally, the remaining $M-(K-2) D$ rows are chosen so that they hear only Transmitter $k+K-1$, denoted as $S_{k c}$, which is possible since the common nullspace of $K-2$ cross channels has $M-(K-2) D$ dimensions.

Step 2: A corresponding change of basis is done at the transmitters. Within the $M$-dimensional signal space at Transmitter $k$, there is the $M-(K-2) D$ dimensional subspace which is seen at Receiver $k+1$ at the antennas denoted as $S_{(k+1) c}$. This space is denoted as $X_{k c}$ at Transmitter $k$. The remaining $(K-2) D$ dimensions at Transmitter $k$ correspond to the $K-2$ spaces, each of $D$ dimensions, denoted individually as $X_{k a_{i}}$, that are heard by Receivers $k-i$, at antennas $(k-i) a_{i}$, $\forall i=\{1, \ldots, K-2\}$.

The resulting network connectivity after the change of basis operations is shown in Figure 14.

C.2.b) Outer bound: For the outer bound we will provide enough genie information to Receiver $k$ to allow it to decode all $K$ messages. Receiver $k$ is already guaranteed to decode its desired message. In order for Receiver $k$ to decode the remaining $K-1$ (undesired) messages after it decodes and subtracts the signal from its desired transmitter, it needs sufficiently many linear equations. From Transmitter $k+K-1$ (same as Transmitter $k-1$ since indices are interpreted modulo $K$ ), the genie information given to Receiver $k$, which is denoted as $\mathcal{G}_{k, k+K-1}$, is comprised of (noisy versions of) signals $X_{(k+K-1) a_{i}}^{n}, \forall i=\{1,2, \cdots, K-2\}$. Thus, in addition to $S_{k c}$ (equivalently $X_{(k+K-1) c}^{n}$ ) that is already available, the genie information provides Receiver $k$ enough equations to decode and subtract the message from Transmitter $k+K-1$. For each of the remaining $K-2$ transmitters, i.e., transmitters $k+1, k+2, \cdots, k+K-2$, the genie provides the $M-D$ dimensional signals not seen by Receiver $k$, e.g., from Transmitter $k+1$, the genie provides $\mathcal{G}_{k, k+1}$ which is comprised of $X_{(k+1) a_{i}}^{n} \forall i=\{2,3, \cdots, K-2\}$ as well as $X_{(k+1) c}^{n}$. From Transmitter $k+2$, the genie provides $\mathcal{G}_{k, k+2}$ which is comprised of $X_{(k+2) a_{i}}^{n} \forall i=\{1,3,4, \cdots, K-2\}$ as well as $X_{(k+2) c}^{n}$, and so on. This allows Receiver $k$ to decode all $K$ messages, and produces the following outer bound.

$$
\begin{aligned}
n R_{\Sigma} \leq & I\left(\bar{Y}_{k}^{n}, \mathcal{G}_{k, k+1}, \cdots, \mathcal{G}_{k, k+K-1}\right. \\
& \left.W_{1}, W_{2}, \cdots, W_{K}\right)+o(n) \\
\leq & M n \log \rho+h\left(\mathcal{G}_{k, k+1}, \cdots, \mathcal{G}_{k, k+K-1} \mid \bar{Y}_{k}^{n}, W_{k}\right) \\
& +n o(\log \rho)+o(n) \\
\leq & M n \log \rho+h\left(\mathcal{G}_{k, k+K-1} \mid X_{(k+K-1) c}^{n}\right) \\
& +h\left(\mathcal{G}_{k, k+1} \mid X_{(k+1) a_{1}}^{n}\right)+h\left(\mathcal{G}_{k, k+2} \mid X_{(k+2) a_{2}}^{n}\right) \\
& +\cdots+h\left(\mathcal{G}_{k, k+K-2} \mid X_{(k+K-2) a_{K-2}}^{n}\right) \\
& +n o(\log \rho)+o(n) \\
\leq & M n \log \rho+n\left(R_{\Sigma}-R_{k}\right) \\
& -h\left(X_{(k+K-1) c}^{n}\right)-h\left(X_{(k+1) a_{1}}^{n}\right) \\
& -h\left(X_{(k+2) a_{2}}^{n}\right)-\cdots-h\left(X_{(k+K-2) a_{K-2}}^{n}\right) \\
\Rightarrow n R_{k} \leq & M n \log \rho+n o(\log \rho)+o(n) \\
& -h\left(X_{(k+K-1) c}^{n}\right)-h\left(X_{(k+1) a_{1}}^{n}\right)-h\left(X_{(k+2) a_{2}}^{n}\right) \\
& -\cdots-h\left(X_{(k+K-2) a_{K-2}}^{n}\right)
\end{aligned}
$$

See also [20] for additional insights into this approach for DoF outer bounds. Advancing user indices and adding the bounds for all receivers, we have:

$$
\begin{aligned}
& n R_{\Sigma} \leq K M n \log \rho+n o(\log \rho)+o(n) \\
&-\sum_{k=1}^{K}\left(h\left(X_{k c}^{n}\right)-h\left(X_{k a_{1}}^{n}\right)-h\left(X_{k a_{2}}^{n}\right)\right. \\
&\left.-\cdots-h\left(X_{k a_{K-2}}^{n}\right)\right) \\
& \leq K M n \log \rho+n o(\log \rho)+o(n) \\
&-\sum_{k=1}^{K} h\left(X_{k a_{1}}^{n}, X_{k a_{2}}^{n}, \cdots, X_{k a_{K-2}}^{n}, X_{k c}^{n}\right) \\
& \leq K M n \log \rho+n o(\log \rho)+o(n) \\
&-\sum_{k=1}^{K} h\left(X_{k}^{n}\right) \\
& n R_{\Sigma} \leq K M n \log \rho+n o(\log \rho)+o(n)-n R_{\Sigma} \\
& \Rightarrow n R_{\Sigma} \leq \frac{K M}{2} n \log \rho+n o(\log \rho)+o(n)
\end{aligned}
$$

Thus, the sum DoF are bounded above by $K M / 2$ and the DoF per user are bounded above as $d \leq \frac{M}{2}$ when $(K-2) D \leq M<(K-1) D$.

C.3) $K$-User Channel With $(\mathbf{K}-\mathbf{2}) \mathbf{D}>\mathbf{M}$ : Here, DoF per user are outer bounded by $\frac{M}{2}$ since $\frac{M}{2}$ is the outer bound per user for a similar channel with only $K-1$ users. Adding a user 


\begin{tabular}{r|r|c|}
\hline$\left|X_{1 a_{1}}\right|=D$ & $X_{1 a_{1}}$ & $\circ$ \\
\hline & $\vdots$ & $\vdots$ \\
\hline$\left|X_{1 a_{K-2}}\right|=D$ & $X_{1 a_{K-2}}$ & $\circ$ \\
\hline$\left|X_{1 c}\right|=M-(K-2) D$ & $X_{1 c}$ & $\circ$ \\
\hline
\end{tabular}

\begin{tabular}{c|l|l}
\hline$\circ$ & $S_{1 a_{1}}\left(X_{2 a_{1}}, X_{K}\right)$ & $\left|S_{1 a_{1}}\right|=D$ \\
\hline$\vdots$ & $\vdots$ & \\
\hline$\circ$ & $S_{1 a_{K-2}}\left(X_{(K-1) a_{K-2}}, X_{K}\right)$ & $\left|S_{1 a_{K-2}}\right|=D$ \\
\hline$\circ$ & $S_{1 c}\left(X_{K c}\right)$ & $\left|S_{1 c}\right|=M-(K-2) D$ \\
\hline
\end{tabular}

\begin{tabular}{r|r|c|}
\hline$\left|X_{2 a_{1}}\right|=D$ & $X_{2 a_{1}}$ & $\circ$ \\
\hline & $\vdots$ & $\vdots$ \\
\hline$\left|X_{2 a_{K-2}}\right|=D$ & $X_{2 a_{K-2}}$ & $\circ$ \\
\hline$\left|X_{2 c}\right|=M-(K-2) D$ & $X_{2 c}$ & $\circ$ \\
\hline
\end{tabular}

\begin{tabular}{|c|l|l}
\hline$\circ$ & $S_{2 a_{1}}\left(X_{3 a_{1}}, X_{1}\right)$ & $\left|S_{2 a_{1}}\right|=D$ \\
\hline$\vdots$ & $\vdots$ & \\
\hline$\circ$ & $S_{2 a_{K-2}}\left(X_{K a_{K-2}}, X_{1}\right)$ & $\left|S_{2 a_{K-2}}\right|=D$ \\
\hline$\circ$ & $S_{2 c}\left(X_{1 c}\right)$ & $\left|S_{2 c}\right|=M-(K-2) D$ \\
\hline
\end{tabular}

\begin{tabular}{r|r|c|}
\hline$\left|X_{3 a_{1}}\right|=D$ & $X_{3 a_{1}}$ & $\circ$ \\
\hline & $\vdots$ & $\vdots$ \\
\hline$\left|X_{3 a_{K-2}}\right|=D$ & $X_{3 a_{K-2}}$ & $\circ$ \\
\hline$\left|X_{3 c}\right|=M-(K-2) D$ & $X_{3 c}$ & $\circ$ \\
\hline
\end{tabular}

\begin{tabular}{|c|l|l}
\hline$\circ$ & $S_{3 a_{1}}\left(X_{4 a_{1}}, X_{2}\right)$ & $\left|S_{3 a_{1}}\right|=D$ \\
\hline$\vdots$ & $\vdots$ & \\
\hline$\circ$ & $S_{3 a_{K-2}}\left(X_{1 a_{K-2}}, X_{2}\right)$ & $\left|S_{3 a_{K-2}}\right|=D$ \\
\hline$\circ$ & $S_{3 c}\left(X_{2 c}\right)$ & $\left|S_{3 c}\right|=M-(K-2) D$ \\
\hline
\end{tabular}

\begin{tabular}{r|r|c|}
\hline$\left|X_{K a_{1}}\right|=D$ & $X_{K a_{1}}$ & $\circ$ \\
\hline & $\vdots$ & $\vdots$ \\
\hline$\left|X_{K a_{K-2}}\right|=D$ & $X_{K a_{K-2}}$ & $\circ$ \\
\hline$\left|X_{K c}\right|=M-(K-2) D$ & $X_{K c}$ & $\circ$ \\
\hline
\end{tabular}

\begin{tabular}{|c|c|c|}
\hline 0 & $S_{K a_{1}}\left(X_{1 a_{1}}, X_{K-1}\right)$ & $\left|S_{K a_{1}}\right|=D$ \\
\hline$\vdots$ & $\vdots$ & \\
\hline O & $S_{K a_{K-2}}\left(X_{(K-2) a_{K-2}}, X_{K-1}\right)$ & $\left|S_{K a_{K-2}}\right|=D$ \\
\hline$\circ$ & $S_{K c}\left(X_{(K-1) c}\right)$ & $\left|S_{K c}\right|=M-(K-2) D$ \\
\hline
\end{tabular}

Fig. 14. Outer bound: $K$-user rank deficient interference channel, $(K-2) D \leq M<(K-1) D$.

cannot increase the DoF per user. This is a recursive argument, in the sense that the 4-user channel uses the known outer bound of $\frac{M}{2}$ for the 3-user channel for the region $2 D>M$. Similarly, the 5-user channel uses the known outer bound of $\frac{M}{2}$ for the 4-user channel for the region $3 D>M$ and so on.

For example, let us consider the 4-user rank deficient channel in which sum of 3 cross channel ranks $3 D>\frac{3 M}{2}$. Within this channel, the 3-user interference channel corresponding to first 3 users has sum of cross channel ranks $2 D>M$, a region for which outer bound per user is known to be $\frac{M}{2}$. Adding the fourth user to this 3-user network cannot increase the DoF per user. Hence $\frac{M}{2}$ is also an outer bound for the 4-user rank deficient channel. This argument is readily extended to $K$ user channels to show that $\frac{M}{2}$ is the DoF outer bound per user if $(K-2) D>M$.

$$
\frac{\mathrm{DoF}}{K} \leq \min \left(D_{0}, M-\frac{\min (M,(K-1) D)}{2}\right)
$$

Combining DoF outer bound results of all 3 regions along with min-cut bound of $D_{0}$ (direct channel rank), we get above outer bound on DoF per user for $K$-user rank deficient interference channel, as stated in Theorem 3.

\section{CONCLUSIONS}

Spatial dependencies often arise in MIMO interference networks, that impact their signaling dimensions. In this work, we studied spatial dependencies that are manifested as rank deficiencies of the MIMO channel matrices. Implications of rank deficiencies on DoF of MIMO interference networks were explored, involving either asymptotic or non-asymptotic interference alignment schemes. One of the key observations is that rank deficiencies of the cross channels cannot hurt and could even improve the DoF, while rank deficiencies of the direct channels cannot help and could hurt. 2-user and 3-user interference channels were studied involving non-asymptotic schemes for both constant and time-varying channels. While the 2-user channel only requires zero forcing, the 3-user channel involves both zero-forcing and interference alignment. For the 3-user channel with rank deficiencies, although there is more opportunity for zero-forcing and less opportunity for interference alignment, the increased opportunity for zero-forcing apparently more than compensates for the lost opportunity in interference alignment. Additional challenges are involved for the $K$-user interference channels with rank deficiencies.

Both asymptotic interference alignment $(\mathrm{CJ})$ and ergodic alignment schemes were studied in the context of $K$-user rank deficient interference channels with time-varying channel coefficients $(K>3)$. For the $K$-user interference channel with individual channels of size $M \times M$ being rank deficient, optimal DoF per user was characterized as $\min \left(D_{0}, M-\frac{\min (M,(K-1) D)}{2}\right)$ where $D_{0}$ is the rank of direct channels, and $D$ is the rank of cross channels. When using CJ scheme, one of the remarkable aspects is that rank deficiencies in cross channels lead to columns of the precoding matrix being linearly dependent, however, by discarding those linearly dependent columns, DoF per user can be made arbitrarily close to $\frac{1}{2}$. We expect that the insights presented in this work 
would serve as stepping stones to translating DoF result to $K$-user rank deficient interference channels with constant channel coefficients. It could be noted that the achievable scheme involves joint processing of signals (one-sided decomposition) at the receivers, for both ergodic and asymptotic interference alignment schemes. This is due to presence of spatial dependencies among certain direct channels and cross channels in the fully decomposed network, because of rank deficient direct channels in the original network. While joint processing is sufficient to achieve optimal DoF using either ergodic or CJ scheme, whether it is also necessary is an intriguing open problem.

The single user MIMO channel problem with full decomposition (no joint processing) is equivalent to the problem wherein the overall channel of a SISO interference channel is rank deficient. In a $K$-user SISO interference channel, the overall $K \times K$ channel could be rank deficient, say of rank $D$. This could arise because of network topology, wherein relays with $D$ antennas listen to signals from $K$ transmitters and amplify and forward the signals to $K$ receivers. Study of alignment feasibility for multiple unicast sessions with similar multihop network topologies is another relevant problem, related to the problem studied in [16] for 3 users.

\section{ACKNOWLEDGEMENTS}

The authors would like to thank Zeng et al. [25] for pointing out a problem in the achievable scheme for Theorem 2, in the Globecom version of this paper. S. R. Krishnamurthy would also like to thank Chenwei Wang and Hua Sun for insightful discussions during the course of this work.

\section{REFERENCES}

[1] Y. Birk and T. Kol, "Informed-source coding-on-demand (ISCOD) over broadcast channels," in Proc. 17th Annu. Joint Conf. IEEE Comput. Commun. Soc. (INFOCOM), vol. 3. Mar./Apr. 1998, pp. 1257-1264.

[2] M. A. Maddah-Ali, A. S. Motahari, and A. K. Khandani, "Communication over MIMO X channels: Interference alignment, decomposition, and performance analysis," IEEE Trans. Inf. Theory, vol. 54, no. 8, pp. 3457-3470, Aug. 2008.

[3] S. A. Jafar and S. Shamai, "Degrees of freedom region of the MIMO X channel," IEEE Trans. Inf. Theory, vol. 54, no. 1, pp. 151-170, Jan. 2008.

[4] V. R. Cadambe and S. A. Jafar, "Interference alignment and degrees of freedom of the $K$-user interference channel," IEEE Trans. Inf. Theory, vol. 54, no. 8, pp. 3425-3441, Aug. 2008.

[5] S. Jafar, "Interference alignment: A new look at signal dimensions in a communication network," in Foundations and Trends in Communication and Information Theory. Norwell, MA, USA: NOW Pub., 2011, pp. $1-136$.

[6] S. A. Jafar and M. J. Fakhereddin, "Degrees of freedom for the MIMO interference channel," IEEE Trans. Inf. Theory, vol. 53, no. 7, pp. 2637-2642, Jul. 2007.

[7] C. Wang, T. Gou, and S. A. Jafar, "Subspace alignment chains and the degrees of freedom of the three-user MIMO interference channel," IEEE Trans. Inf. Theory, vol. 60, no. 5, pp. 2432-2479, May 2014.

[8] G. Bresler, D. Cartwright, and D. Tse, "Geometry of the 3-user MIMO interference channel," in Proc. 49th Annu. Allerton Conf. Commun., Control, Comput. (Allerton), Sep. 2011, pp. 1264-1271.

[9] K. Gomadam, V. R. Cadambe, and S. A. Jafar, "A distributed numerical approach to interference alignment and applications to wireless interference networks," IEEE Trans. Inf. Theory, vol. 57, no. 6, pp. 3309-3322, Jun. 2011.

[10] C. M. Yetis, T. Gou, S. A. Jafar, and A. H. Kayran, "On feasibility of interference alignment in MIMO interference networks," IEEE Trans. Signal Process., vol. 58, no. 9, pp. 4771-4782, Sep. 2010.
[11] V. R. Cadambe and S. A. Jafar, "Interference alignment and the degrees of freedom of wireless $X$ networks," IEEE Trans. Inf. Theory, vol. 55, no. 9, pp. 3893-3908, Sep. 2009.

[12] V. S. Annapureddy, A. El Gamal, and V. V. Veeravalli, "Degrees of freedom of interference channels with CoMP transmission and reception," IEEE Trans. Inf. Theory, vol. 58, no. 9, pp. 5740-5760, Sep. 2012.

[13] C. Wang, S. A. Jafar, S. Shamai, and M. Wigger, "Interference, cooperation and connectivity-A degrees of freedom perspective," in Proc. IEEE ISIT, Jul./Aug. 2011, pp. 430-434.

[14] C. Wang and A. Sezgin, "Degrees of freedom of the interference channel with a cognitive helper," IEEE Commun. Lett., vol. 17, no. 5 , pp. 920-923, May 2013.

[15] V. R. Cadambe, S. A. Jafar, H. Maleki, K. Ramchandran, and C. Suh, "Asymptotic interference alignment for optimal repair of MDS codes in distributed storage," IEEE Trans. Inf. Theory, vol. 59, no. 5, pp. 2974-4987, May 2013.

[16] C. Meng, A. Das, A. Ramakrishnan, S. A. Jafar, A. Markopoulou, and S. Vishwanath, "Precoding-based network alignment for three unicast sessions," IEEE Trans. Inf. Theory, 2014, Arxiv ID 1305.0868, doi: 10.1109/TIT.2014.2368556.

[17] A. S. Motahari, S. O. Gharan, M. Maddah-Ali, and A. K. Khandani. (2009). "Real interference alignment: Exploiting the potential of single antenna systems." [Online]. Available: http://arxiv.org/abs/0908.2282

[18] L. Ke, A. Ramamoorthy, Z. Wang, and H. Yin, "Degrees of freedom region for an interference network with general message demands," IEEE Trans. Inf. Theory, vol. 58, no. 6, pp. 3787-3797, Jun. 2012.

[19] A. Ghasemi, A. S. Motahari, and A. K. Khandani, "Interference alignment for the K user MIMO interference channel," in Proc. Int. Symp. Inf. Theory (ISIT), Jun. 2010, pp. 360-364.

[20] C. Wang, H. Sun, and S. A. Jafar. (2014). "Genie chains: Exploring outer bounds on the degrees of freedom of MIMO interference networks." [Online]. Available: http://arxiv.org/abs/1404.2258

[21] H. Sun and S. A. Jafar, "Degrees of freedom of MIMO $X$ networks: Spatial scale invariance and one-sided decomposability," IEEE Trans. Inf. Theory, vol. 59, no. 12, pp. 8377-8385, Dec. 2013.

[22] G. Sridharan and W. Yu. (2013). "Degrees of freedom of MIMO cellular networks: Decomposition and linear beamforming design." [Online]. Available: http://arxiv.org/abs/1312.2681

[23] S. H. Chae and S.-Y. Chung, "On the degrees of freedom of rank deficient interference channels," in Proc. IEEE Int. Symp. Inf. Theory (ISIT), Jul./Aug. 2011, pp. 1367-1371.

[24] S. R. Krishnamurthy and S. A. Jafar, "Degrees of freedom of 2-user and 3-user rank-deficient MIMO interference channels," in Proc. IEEE Global Commun. Conf. (GLOBECOM), Dec. 2012, pp. 2462-2467.

[25] Y. Zeng, X. Xu, Y. L. Guan, and E. Gunawan. (2012). "On the achievable degrees of freedom for the 3-user rank-deficient MIMO interference channel." [Online]. Available: http://adsabs. harvard.edu/abs/2012arXiv1211.4198Z

[26] A. Agustin and J. Vidal, "Degrees of freedom of the rank-deficient MIMO X channel," in Proc. IEEE Inf. Theory Workshop (ITW), Sep. 2013, pp. 1-5.

[27] S. A. Jafar, "Blind interference alignment," IEEE J. Sel. Topics Signal Process., vol. 6, no. 3, pp. 216-227, Jun. 2012.

[28] B. Nazer, M. Gastpar, S. A. Jafar, and S. Vishwanath, "Ergodic interference alignment," IEEE Trans. Inf. Theory, vol. 58, no. 10, pp. 6355-6371, Oct. 2012.

[29] M. Zamanighomi and Z. Wang, "Multiple-antenna interference channel with receive antenna joint processing and real interference alignment," in Proc. ISIT, Jul. 2013, pp. 564-568.

[30] J. T. Schwartz, "Fast probabilistic algorithms for verification of polynomial identities," J. Assoc. Comput. Mach., vol. 27, no. 4, pp. 701-717, Oct. 1980

[31] R. Zippel, "Probabilistic algorithms for sparse polynomials," in Proc. Int. Symp. Symbolic Algebraic Comput. (EUROSAM), 1979, pp. 216-226.

Sundar R. Krishnamurthy (S'12) received his B. E. (Hons.) from BITS, Pilani, India, in 2003, M.S. and Ph.D. from Univ. of California, Irvine (UC Irvine), USA, in 2011 and 2014 respectively, all in Electrical Engineering. His industry experience includes positions at Qualcomm Research, Samsung Electronics and Intel Corporation. His research interests include information theory and signal processing for communications. 
Abinesh Ramakrishnan (S'12) received his B.E. degree in Electronics and Communications Engineering from the Madras Institute of Technology, Anna University, India in 2008 and his Master's degree from EECS department at the University of California, Irvine in 2010. He is currently a Ph.D. candidate in the EECS department at UCI. He was an intern at Huawei US R\&D Center during Fall 2013. His research interests include network coding, information theory, interference alignment, index coding, distributed storage and content delivery.
Syed Ali Jafar (S'99-M'04-SM'09-F'14) received his B. Tech. from IIT Delhi, India, in 1997, M.S. from Caltech, USA, in 1999, and Ph.D. from Stanford, USA, in 2003, all in Electrical Engineering. His industry experience includes positions at Lucent Bell Labs, Qualcomm Inc. and Hughes Software Systems. He is currently an Associate Professor and Associate Chair for Graduate Studies in the Department of Electrical Engineering and Computer Science at the University of California Irvine, Irvine, CA USA. His research interests include multiuser information theory and wireless communications.

Dr. Jafar received the NSF CAREER award in 2006, the ONR Young Investigator Award in 2008, the Information Theory Society paper award in 2009, the School of Engineering Maseeh Outstanding Research Award in 2010, an IEEE GLOBECOM CTS Best Paper Award in 2012 and an IEEE Communication Society Best Tutorial Paper Award in 2013. Dr. Jafar received the UC Irvine EECS Professor of the Year award five times, in 2006, 2009, 2011, 2012, and 2014, from the Engineering Students Council and the Teaching Excellence Award in 2012 from the School of Engineering. He was a University of Canterbury Erskine Fellow in 2010 and is an IEEE Communications Society Distinguished Lecturer for 2013-2014. Dr. Jafar is a Thomson Reuters Highly Cited Researcher. He served as Associate Editor for IEEE TRANSACTIONS ON COMMUNICATIONS 2004-2009, for IEEE COMMUNICATIONS LETTERS 2008-2009 and for IEEE TRANSACTIONS ON INFORMATION THEORY 2009- 2012. 